\title{
Holographic entanglement entropy with momentum relaxation
}

\author{
Kyung Kiu Kim ${ }^{1, a}$, Chanyong Park ${ }^{2,3,4, b}$, Jung Hun Lee ${ }^{2,3, c} \mathbb{D}_{\mathbb{D}}$, Byoungjoon Ahn ${ }^{5, \mathrm{~d}}$ \\ ${ }^{1}$ Department of Physics, Sejong University, Seoul 05006, South Korea \\ 2 Department of Physics and Photon Science, Gwangju Institute of Science and Technology, Gwangju 61005, South Korea \\ ${ }^{3}$ Asia Pacific Center for Theoretical Physics, Pohang 790-784, South Korea \\ ${ }^{4}$ Department of Physics, Postech, Pohang 790-784, South Korea \\ ${ }^{5}$ Department of Physics, College of Science, Yonsei University, Seoul 120-749, South Korea
}

Received: 8 January 2019 / Accepted: 18 April 2019 / Published online: 30 April 2019

(C) The Author(s) 2019

\begin{abstract}
We studied the holographic entanglement entropy for a strip and sharp wedge entangling regions in momentum relaxation systems. In the case of strips, we found analytic and numerical results for the entanglement entropy and examined the effect of the electric field on the entanglement entropy. We also studied the entanglement entropy of wedges and confirmed that there is a linear change due to the electric field. In this case, we showed that the entanglement entropy change is interestingly proportional to the thermoelectric conductivity which is a measurable quantity. Also, we discussed comparable calculations with ABJM theory and we suggested an experiment for our result.
\end{abstract}

\section{Introduction}

Entanglement entropy plays a prominent role in various areas of physics such as quantum field theory, gravity theory and condensed matter physics. However, it is very difficult to evaluate this amount because of non-local property. Fortunately, an effective way to calculate entanglement entropy has recently been developed by Ryu and Takayanagi [1]. Since this method is based on AdS/CFT correspondence [2,3] which relates gravitational systems to quantum field theories, it may shed a light on understanding quantum gravity [4-6]. Apart from this, the method is useful to study various interesting cases. For examples, it has been used to distinguish different phases e.g., [7-9] and to study thermalization under quantum quenches e.g., [10-13]. Further studies with the method show us new universal properties of generic CFT e.g., [14-18]. Even for a particular non-conformal field the-

\footnotetext{
a e-mail: kimkyungkiu@ @ejong.ac.kr

be-mail: cyong21@gist.ac.kr

c e-mail: junghun.lee@gist.ac.kr

de-mail: bjahn@yonsei.ac.kr
}

ory, it turns out that Ryu and Takayanagi's proposal is still useful by considering a top-down study on the field theory and the corresponding supergravity solution [19-25].

Entanglement entropy is a very important quantity but, in most cases, it is difficult to calculate. It is also unclear whether this amount can be measured easily. ${ }^{1}$ Therefore, it is hard to tell if Ryu and Takayanagi's method is valid in various cases. In this study, we try to find examples where entanglement entropy changes can be represented by other readily measurable physical quantities. Like thermal entropy, the entanglement entropy responses to other external sources. One of such sources which can be produced in a laboratory is the electric field. Thus we will investigate how the entanglement entropy changes under the electric field using holographic methods.

Accordingly, to demonstrate a system in the electric field holographically, we need to consider a gravity dual describing the system. The Ryu-Takayanagi's formula and the gravity dual allow us to study the response of entanglement entropy to the electric field. For the sake of simplicity, we introduce a time-independent electric field, i.e, DC electric field. To realize such a situation, we take the momentum relaxation into account. This is because infinite DC currents can occur without the momentum relaxation. The gravity duals have been developed to study holographic DC conductivities in the context of AdS/CMT e.g., [26-46] and some interesting time-dependent extensions e.g., [47-50]. Among these backgrounds, we adopted a background geometry of an axion model, where a scalar field breaks translation invariance explicitly [28].

In the absence of the bulk gauge field, an analytic form of holographic entanglement entropy with small momentum relaxation was studied in [51]. In this work, we performed

\footnotetext{
${ }^{1}$ Recently quantum purity, Rényi entanglement entropy and mutual information was measured in experiments [73].
} 
analytic and numerical studies on the strip entangling region in the presence of the bulk electric field, that is dual to the density and the external electric field in the boundary system. We obtained new analytic expressions of the entanglement entropy in the very narrow strip limit. Since our study is about $2+1$ dimensional field theory systems, one may compare the result to a field theoretic calculation qualitatively. So, we suggest a comparable situation in the ABJM theory [52]. Also, we provide numerical results without taking any limit. Our numerical results reproduce the earlier work [53] and extend its numerical study. This calculation shows that entanglement entropy becomes larger with more charge density and stronger momentum relaxation. We will discuss the physical implication for the extended parameter space.

Furthermore, we studied how the strip type entanglement entropy changes by turning on the constant electric field. And we found that the minimal surface anchored to the strip is tilted by the electric field and the deformation is proportional to the thermoelectric conductivity $\bar{\alpha}$ in the conductivity tensor. This is a quite interesting result because the thermoelectric conductivity is a clearly measurable quantity. However, the entanglement entropy changes from the deformation do not appear in the linear order of the electric field. To see the effect, we need to consider the quadratic order gravity dual but it is beyond our present work.

Instead we consider the wedge type entangling region whose symmetric axis is not orthogonal to the electric field . Finally, we took a sharp wedge limit and obtained the entanglement entropy changes at the linear level of the electric field. And we confirmed that the response to the electric field is proportional to the anticipated thermoelectric conductivity $\bar{\alpha}$. This result is also remarkable because the thermoelectric conductivity or the Nernst signal could reflect the existence of the quantum critical point even in the normal phase of the superconductor $[41,54,55,76]$. Thus it is suggested that the entanglement entropy may be associated with the quantum critical point.

This paper is organized as follows: In Sect. 2 we introduce the bulk solution dual to a momentum relaxation system with the electric field and summarize how the conductivities can be related to the bulk fields. In Sect. 3, we study on the holographic entanglement entropy for the strip entangling region. In Sect. 4, we consider the holographic entanglement entropy for the wedge region. In Sect. 5. we conclude our work.

\section{A gravity dual of momentum relaxation with electric field}

In this section we review a gravity dual to a momentum relaxation system in the electric field. The geometry has been discussed to study finite DC conductivity e.g., [28,40]. The momentum relaxation in physical systems is essential to obtain finite DC conductivity. Let us start with a simple model with momentum relaxation:

$S=\frac{1}{16 \pi G} \int d^{4} x \sqrt{-g}\left(R+6-\frac{1}{4} F^{2}-\frac{1}{2} \sum_{\mathcal{I}=1}^{2}\left(\partial \chi_{\mathcal{I}}\right)^{2}\right)$.

This system admits a black brane solution as follows:

$d s^{2}=-U(r) d t^{2}+r^{2}\left(d x^{2}+d y^{2}\right)+\frac{d r^{2}}{U(r)}$,

$A=q\left(\frac{1}{r_{h}}-\frac{1}{r}\right) d t, \chi_{\mathcal{I}}=(\beta x, \beta y)$,

with $U(r)=\left(r^{2}-\frac{\beta^{2}}{2}-\frac{M}{r}+\frac{q^{2}}{4 r^{2}}\right) \cdot{ }^{2}$ The massless scalar field, $\chi_{\mathcal{I}}$, is associated with momentum relaxation, breaking the translational symmetry and inducing finite DC conductivity. To see this, let us turn on the external electric field and other related fields along the $x$-direction

$\delta A_{x}=-E_{x} t+a_{x}(r), \quad \delta g_{t x}=r^{2} h_{t x}(r)$,
$\delta g_{r x}=r^{2} h_{r x}(r), \quad \delta \chi_{x}=\psi_{x}(r)$,

where $E_{x}$ corresponds to the external field and other fluctuations are dual to the electric current and the heat current in the boundary system.

Then, the linearized equations of motion are given as follows :

$$
\begin{aligned}
& h_{t x}^{\prime \prime}(r)+\frac{4 h_{t x}^{\prime}(r)}{r}-\frac{\beta^{2} h_{t x}(r)}{r^{2} U(r)}+\frac{q a_{x}^{\prime}(r)}{r^{4}}=0, \\
& \psi_{x}^{\prime}(r)-\beta h_{r x}(r)-\frac{q E_{x}}{\beta r^{2} U(r)}=0, \\
& a_{x}^{\prime \prime}(r)+\frac{U^{\prime}(r) a_{x}^{\prime}(r)}{U(r)}+\frac{q h_{t x}^{\prime}(r)}{U(r)}=0, \\
& \psi_{x}^{\prime \prime}(r)+\left(\frac{U^{\prime}(r)}{U(r)}+\frac{2}{r}\right) \psi_{x}^{\prime}(r) \\
& -\beta h_{r x}^{\prime}(r)-\beta\left(\frac{U^{\prime}(r)}{U(r)}+\frac{2}{r}\right) h_{r x}(r)=0,
\end{aligned}
$$

where Eq. (5) comes from the Einstein equation, and Eq. (6) and (7) are the equations of motion of the matter fields. Note that all the equations above are not independent because Eq. (7) can be obtained by rearranging the other equations. As a consequence, only three equations in (5) and (6) determine the profile of the fluctuations for given boundary conditions. Another thing we need to know is that $h_{r x}$ is not a dynamical field and it plays a role of a Lagrange multiplier. In fact, this is a shift vector in an ADM decomposition along the holographic radial direction. We will discuss the gauge fixing later.

\footnotetext{
${ }^{2}$ We set the AdS radius to 1 and $F=d A$.
} 
The electric current and the heat current using gauge/ gravity duality are given by

$$
\begin{aligned}
J^{x} & =\lim _{r \rightarrow \infty} \mathcal{J}(r), \\
Q^{x} & =T^{t x}-\mu J^{x}=\lim _{r \rightarrow \infty} \mathcal{Q}(r),
\end{aligned}
$$

where

$$
\begin{aligned}
& \mathcal{J}(x) \equiv \sqrt{-g} F^{x r}=U(r) a_{x}^{\prime}(r)+q h_{t x}(r), \\
& Q(r) \equiv U^{2}(r)\left(\frac{\delta g_{t x}}{U(r)}\right)^{\prime}-A_{t}(r) \mathcal{J}(r) .
\end{aligned}
$$

In addition, it can be easily checked that $\mathcal{J}(r)$ and $\mathcal{Q}(r)$ remain as constants along the holographic radial direction $r$ by using the equations of motion (5-7). Therefore, the boundary currents can be computed at the horizon $r=r_{h}$

$$
J^{x}=\lim _{r \rightarrow r_{h}} \mathcal{J}(r), \quad Q^{x}=\lim _{r \rightarrow r_{h}} \mathcal{Q}(r),
$$

Near the horizon the fields behave as

$$
\begin{aligned}
a_{x} & \sim-\frac{E_{x} \log \left(r-r_{h}\right)}{4 \pi T}+a_{x}^{(0)}+\mathcal{O}\left(r-r_{h}\right), \\
h_{t x} & \sim h_{t x}^{(0)}+\mathcal{O}\left(r-r_{h}\right), \\
h_{r x} & \sim \frac{\mathbb{H}_{r x}}{r^{2} U(r)}+h_{r x}^{(0)}+\mathcal{O}\left(r-r_{h}\right), \\
\psi_{x} & \sim \psi_{x}^{(0)}+\mathcal{O}\left(r-r_{h}\right), \\
U(r) & \sim 4 \pi T\left(r-r_{h}\right)+\cdots,
\end{aligned}
$$

where the Hawking temperature $T$ is given by $T=\frac{U^{\prime}\left(r_{h}\right)}{4 \pi}$ and the logarithmic term appears due to the ingoing boundary condition. By solving the equations of motion near the horizon, one can obtain

$\mathbb{H}_{r x}=r_{h}^{2} h_{t x}^{(0)}, \quad h_{t x}^{(0)}=-\frac{q E_{x}}{\beta^{2} r_{h}^{2}}$.

Using these results, the linear response theory yields the following electric conductivity $\left(\sigma=J^{x} / E_{x}\right)$ and thermoelectric conductivity $\left(\bar{\alpha}=Q^{x} / E_{x}\right)$ :

$$
\begin{aligned}
& \sigma=1-\frac{q h_{t x}^{(0)}}{E_{x}}=1+\frac{\mu^{2}}{\beta^{2}}, \\
& \bar{\alpha}=-\frac{4 \pi r_{h}^{2} h_{t x}^{(0)}}{E_{x}}=\frac{4 \pi q}{\beta^{2}} .
\end{aligned}
$$

So the constant $\mathbb{H}_{r x}$ is given by a combination of physical parameters as $\mathbb{H}_{r x}=-\frac{1}{4 \pi} \bar{\alpha} E_{x}$.

The following sections will consider minimum surfaces in this geometry. It is convenient to use a formal expansion parameter $\lambda$, which will be taken as 1 after the calculation. This trick allows us to write the metric in the following form:

$$
\begin{aligned}
d s^{2}= & -U(r) d t^{2}+\frac{d r^{2}}{U(r)}+r^{2}\left(d x^{2}+d y^{2}\right) \\
& +2 \lambda\left(\delta g_{t x}(r) d t d x+\delta g_{r x}(r) d r d x\right)+\mathcal{O}\left(\lambda^{2}\right) .
\end{aligned}
$$

In addition to the conductivities, other physical quantities such as temperature, energy density, charge density, and entropy density can be read from the geometry as follows:

$$
\begin{aligned}
& T=\frac{U^{\prime}\left(r_{h}\right)}{4 \pi}=\frac{3 r_{h}}{4 \pi}\left(1-\frac{\beta^{2}}{6 r_{h}^{2}}-\frac{q^{2}}{12 r_{h}^{4}}\right), \\
& \epsilon=2 M=\frac{4 r_{h}^{4}-2 r_{h}^{2} \beta^{2}+q^{2}}{2 r_{h}}, \rho=q, s=4 \pi r_{h}^{2} .
\end{aligned}
$$

These quantities characterize the dual system to the unperturbed geometry with $\lambda=0$.

\section{Holographic entanglement entropy in electric field: strip entangling region}

\subsection{Basic setup}

In this subsection, we use the Ryu-Takayanagi formula to obtain entanglement entropy for the strip region. The strip also extends along a direction perpendicular to the electric field. To describe the minimal surface anchored to the strip, we may consider a two dimensional surface with the following map in the target geometry (14):

$t=0, \quad r=r\left(\sigma_{1}\right), \quad x=\sigma_{1}, \quad y=\sigma_{2}$,

where $y$ coordinate indicates the infinitely extending direction. Then, one can find the action for the minimal surface as follows:

$\mathcal{A}=\int_{-L / 2}^{L / 2} d y \int_{-l / 2}^{l / 2} d x \sqrt{\frac{r^{2} r^{\prime 2}}{U(r)}+r^{4}+2 \lambda r^{2} \delta g_{r x} r^{\prime}}$,

where $L$ is the length of the strip along $y$-direction. This is our basic action for the holographic entanglement entropy. Or, if we define another convenient coordinates $z \equiv \frac{1}{l r}$ and $\sigma \equiv x / l$, we can write down the action in terms of $z(\sigma)$ as follows:

$\mathcal{A}=\frac{L}{l} \int_{-1 / 2}^{1 / 2} d \sigma \sqrt{\frac{z^{\prime 2}}{z^{6} f(z)}+\frac{1}{z^{4}}-2 \lambda \frac{z^{\prime}}{z^{4}} \delta g_{r x}(z)}$,

where $f(z)=\frac{1}{z^{2}}-\frac{\tilde{\beta}^{2}}{2}-\tilde{M} z+\frac{\tilde{q}^{2}}{4} z^{2}$ with $\tilde{\beta}=\beta l, \tilde{M}=M l^{3}$ and $\tilde{q}=q l^{2} . r(x)$ can always be recovered by $r(x)=\frac{1}{l z(x / l)}$. 
The Lagrangian in (19) has no explicit dependence of $\sigma$. So there is a conserved quantity $H$ given by

$H=\frac{L}{l} \frac{1-\lambda \delta g_{r x} z^{\prime}}{z \sqrt{\frac{z^{\prime 2}}{f(z)}+z^{2}\left(1-2 \lambda z^{\prime} \delta g_{r x}\right)}}=\frac{L}{l z_{*}^{2}}$,

where $z_{*} \equiv z\left(\sigma_{*}\right)$ is the location of the tip of the minimal surface. Since we are considering regular surfaces, $z^{\prime}(\sigma)$ vanishes at the tip of the surface. From this relation one can find the integrated first order equations of motion as follows :

$$
\begin{aligned}
z^{\prime}= & \pm \frac{\sqrt{f(z)\left(z_{*}^{4}-z^{4}\right)}}{z}- \\
& \lambda \frac{f(z) \delta g_{r x}(z)\left(z_{*}^{4}-z^{4}\right)}{z^{2}}+\mathcal{O}\left(\lambda^{2}\right) \\
= & \pm \frac{\sqrt{f(z)\left(z_{*}^{4}-z^{4}\right)}}{z}+\lambda E_{x} \frac{\bar{\alpha} l^{4}\left(z_{*}^{4}-z^{4}\right)}{4 \pi}+\mathcal{O}\left(\lambda^{2}\right),
\end{aligned}
$$

where we took a gauge $\delta g_{r x}=\frac{\mathbb{H}_{r x}}{r^{2} U(r)}$, which is consistent with the boundary conditions. ${ }^{3}$ Plugging the above equations into (19), we obtained the regularized area for the minimal surface as follows:

$\mathcal{A}_{\text {reg }}^{\text {strip }}=\frac{2 L}{l} \int_{\epsilon / l}^{z_{*}} d z \frac{z_{*}^{2}}{z^{3} \sqrt{f(z)\left(z_{*}^{4}-z^{4}\right)}}$,

where $\epsilon$ is the length scale corresponding to the UV cutoff. Here, one can notice that the effect from the electric field is encoded in the location of the tip $z_{*}$. By using the RyuTakayanagi formula, the entanglement entropy for the strip is:

$S_{E E}^{\text {strip }}=\frac{\mathcal{A}_{r e g}^{\text {strip }}}{4 G_{N}}$,

where $G_{N}$ is the 4-dimensional Newton constant. In order to show the numerical result, we define the following refined function which is $\frac{4 G_{N} l}{L}$ times the finite part of the entanglement entropy up to the minus sign:

$\hat{S}_{R}^{\text {strip }} \equiv \frac{2 l}{\epsilon}-\frac{4 G_{N} l}{L} S_{E E}^{\text {strip }}$.

\footnotetext{
3 In the calculation we took a gauge $h_{r x}=\frac{\mathbb{H}_{r x}}{r^{2} U(r)}$. It is legitimate because $h_{r x}$ plays a role of a Lagrange multiplier at the linear level and the choice satisfies the regularity condition at the horizon and near the boundary of AdS space. Furthermore, our final result in this work depends on the gauge invariant quantities, such as the thermoelectric conductivity $\bar{\alpha}$ and geometric angle $\delta$. For this reason, the gauge fixing is believed to be adequate to consider this calculation. In addition, we speculate that the gauge fixing would be clearer if we consider the second order effect of the electric field on the geometry. This is because additional constraints must be considered for $g_{r x}$.
}

Now, let us look at the equation of motion for the minimal surface. The equation of motion can be derived from the action (19) and the solution is expanded in terms of $\lambda$ as follows :

$z(\sigma)=z_{0}(\sigma)+\lambda z_{1}(\sigma)+\mathcal{O}\left(\lambda^{2}\right)$.

Then the equation of motion for each order is given by

$$
\begin{aligned}
z_{0}^{\prime \prime} & -\frac{z_{0}^{\prime 2} f^{\prime}\left(z_{0}\right)}{2 f\left(z_{0}\right)}+2 z_{0} f\left(z_{0}\right)+\frac{z_{0}^{\prime 2}}{z_{0}}=0 \\
z_{1}^{\prime \prime} & +\frac{z_{1} f^{\prime}\left(z_{0}\right)^{2} z_{0}^{\prime 2}}{2 f\left(z_{0}\right)^{2}}+2 z_{0} z_{1} f^{\prime}\left(z_{0}\right) \\
& +\frac{z_{0}^{\prime}\left(-z_{1} f^{\prime \prime}\left(z_{0}\right) z_{0}^{\prime}+\delta g_{r x}\left(z_{0}\right) f^{\prime}\left(z_{0}\right) z_{0}^{\prime 2}-2 f^{\prime}\left(z_{0}\right) z_{1}^{\prime}\right)}{2 f\left(z_{0}\right)} \\
& +2 f\left(z_{0}\right)\left(z_{1}-3 z_{0} \delta g_{r x}\left(z_{0}\right) z_{0}^{\prime}\right)+\delta g_{r x}^{\prime}\left(z_{0}\right) z_{0}^{\prime 3} \\
& +\frac{2 z_{0}^{\prime} z_{1}^{\prime}-\delta g_{r x}\left(z_{0}\right) z_{0}^{\prime 3}}{z_{0}}-\frac{z_{1} z_{0}^{\prime 2}}{z_{0}^{2}}=0 .
\end{aligned}
$$

Here, the zeroth order solution is given by an even function from the symmetry of (26). Furthermore, suppose $z_{1}(\sigma)$ is a solution of (27), then one can easily show that $-z_{1}(-\sigma)$ is also a solution. Therefore the solution $z_{1}$ of (27) is an odd function. By using this fact, one can recognize that $z_{*}$ is larger than $z_{0}(0)$ because $z_{0}^{\prime}(0)$ vanishes but there is a non-vanishing $z_{1}^{\prime}(0)$. We found a solution, $z_{0}(\sigma)+\lambda z_{1}(\sigma)$, numerically and plot the solution in Fig. 1.

To find the effect on entanglement entropy, let us come back to (22). In order to get the $z_{*}$, one can consider a shift of the tip along the $\sigma$ direction. It is denoted by $\lambda \Delta$, then the regularity condition of the tip is given by

$z_{0}^{\prime}(\lambda \Delta)+\lambda z_{1}^{\prime}(\lambda \Delta)=0$.

This condition gives us $\Delta=-z_{1}^{\prime}(0) / z_{0}^{\prime \prime}(0)$. Therefore, it turns out that $z_{*}$ is

$$
\begin{aligned}
z_{*} & \sim z_{0}(\lambda \Delta)+\lambda z_{1}(\lambda \Delta) \\
& =z_{0}(0)+\frac{1}{2} \lambda^{2} \Delta^{2} z_{0}^{\prime \prime}(0)+\lambda^{2} \Delta z_{1}^{\prime}(0)+\mathcal{O}\left(\lambda^{3}\right) \\
& =z_{0}(0)-\frac{\lambda^{2} z_{1}^{\prime}(0)^{2}}{2 z_{0}^{\prime \prime}(0)}+\mathcal{O}\left(\lambda^{3}\right) .
\end{aligned}
$$

From this, we found that the tip change is the order of $\lambda^{2}$. However, our background (14), is only valid in the linear order. Therefore, one needs a quadratic background metric to see the effect on entanglement entropy for the strip case. We leave this as a future work.

Although this result doesn't give us the linear variation of entanglement entropy, it is still worth considering the zeroth order calculation as a leading part of another specific kind of entangling region. We will continue our study to a case with the wedge type entangling region in the Sect. 4 . Such a region is regarded as a tail part of the strip case. See the cartoon in Fig. 5. Also, once the very sharp wedge limit is taken 


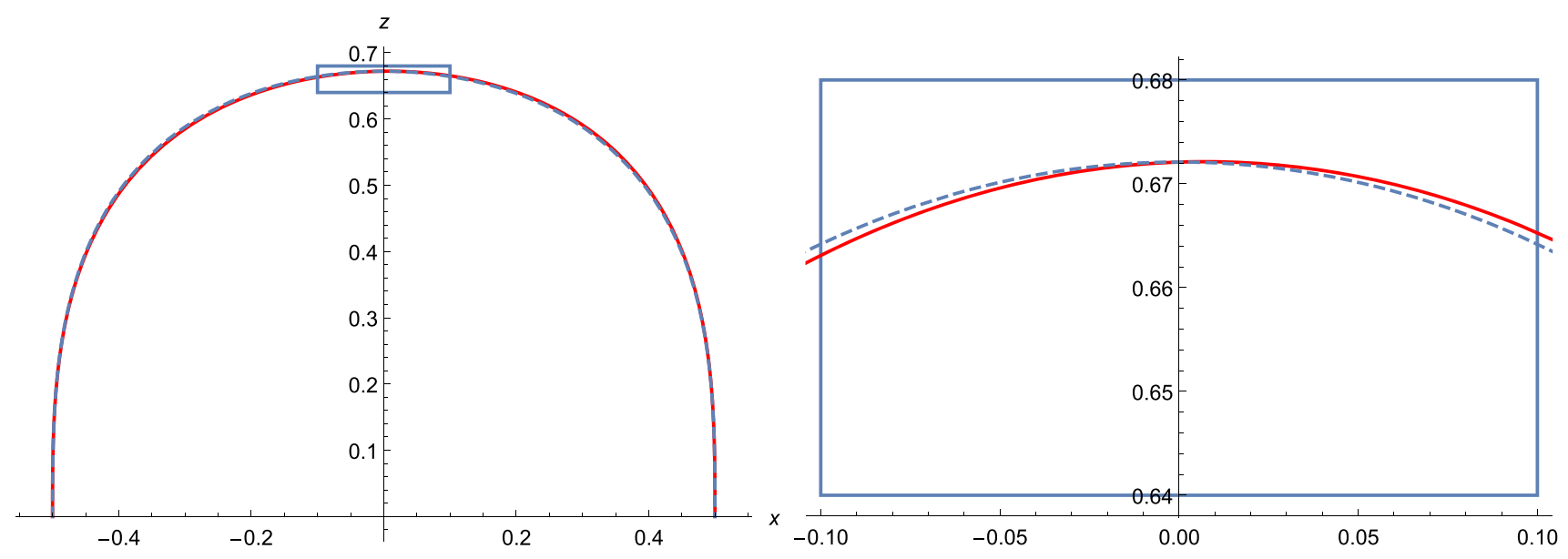

Fig. 1 The intersection of a deformed minimal surface by the electric field (Solid curve) with $\tilde{\beta}=1, \tilde{M}=1$ and $\tilde{q}=1$ : The dotted line denotes the minimal surface before applying the electric field. $\lambda=0.05$ is taken for visualization and the right figure is the enlarged picture for the blue square near the tip

$(\Omega \rightarrow 0$ ), this limit should give rise to the strip result. Due to these reasons, it is desirable to scrutinize the zeroth order calculation for the strip case numerically and analytically in the following subsections.

\subsection{Numerical result for holographic entanglement entropy}

Now we study the holographic entanglement entropy (22) numerically. As examined in the previous subsection, there is no linear variation of the entanglement entropy to the electric field for the strip entangling region. Therefore, in this subsection, we will focus only on numerical results of the entanglement entropy without the electric field .

First, we define the following function to facilitate numerical computation:

$$
\tilde{A}_{0} \equiv A_{\text {reg }}^{(0)} \ell=2 L \int_{\tilde{\epsilon}}^{z_{0}^{*}} d z \frac{z_{0}^{* 2}}{z^{3} \sqrt{f(z)\left(z_{0}^{* 4}-z^{4}\right)}},
$$

where $z_{0}^{*} \equiv z_{0}(0)$ and $\tilde{\epsilon} \equiv \epsilon / l$. For convenience, we replace $\tilde{M}$ with $z_{h}, \tilde{\beta}$ and $\tilde{q}$ using the relation $f\left(z_{h}\right)=0$. Then, the useful expression for $f(z)$ is given by

$f(z)=\frac{\left(z-z_{h}\right)\left(z^{3} \tilde{q}^{2} z_{h}^{3}+2 z^{2} \tilde{\beta}^{2} z_{h}^{2}-4 z_{h}^{2}-4 z z_{h}-4 z^{2}\right)}{4 z^{2} z_{h}^{3}}$.

By adopting a scaled coordinate $u \equiv z / z_{0}^{*}$, we reparameterize $\xi \equiv z_{0}^{*} / z_{h}, \bar{q} \equiv \tilde{q} z_{h}^{2}$ and $\bar{\beta}=\tilde{\beta} z_{h}{ }^{4}$ Then the area function (30) becomes

\footnotetext{
${ }^{4}$ Thus the parameters in terms of the original physical quantities are given by $\xi=\frac{r_{h}}{r_{0}^{*}}, z_{h}=1 /\left(r_{h} l\right), \bar{\beta}=\beta / r_{h}$ and $\bar{q}=q / r_{h}^{2}$.
}

$$
\begin{aligned}
& \tilde{A}_{0}=\frac{2 L}{z_{0}^{*}} \int_{\substack{\tilde{\epsilon} \\
z_{0}^{*}}}^{1} d u \\
& \times \frac{1}{u^{2} \sqrt{\left(1-u^{4}\right)(1-\xi u)\left(1+\xi u+\xi^{2} u^{2}-\frac{1}{2} \bar{\beta}^{2} \xi^{2} u^{2}-\frac{1}{4} \bar{q}^{2} \xi^{3} u^{3}\right)}} .
\end{aligned}
$$

Also, the temperature of the system can be rewritten in terms of the dimensionless parameters as

$$
\begin{aligned}
\tilde{T} \equiv T \ell & =\frac{1}{16 \pi z_{h}}\left(12-\tilde{q}^{2} z_{h}^{4}-2 \tilde{\beta}^{2} z_{h}^{2}\right) \\
& =\frac{\xi}{16 \pi z_{0}^{*}}\left(12-\bar{q}^{2}-2 \bar{\beta}^{2}\right) .
\end{aligned}
$$

In addition, (21) and the regularity determines $z_{0}^{*}$ as follows:

$$
\begin{aligned}
\frac{1}{z_{0}^{*}} & =\int_{0}^{1} d u \\
& \times \frac{2 u^{2}}{\sqrt{\left(1-u^{4}\right)(1-\xi u)\left(1+\xi u+\xi^{2} u^{2}-\frac{1}{2} \bar{\beta}^{2} \xi^{2} u^{2}-\frac{1}{4} \bar{q}^{2} \xi^{3} u^{3}\right)}} .
\end{aligned}
$$

Therefore, for given $\xi, \bar{q}$ and $\bar{\beta}$, the regularized area of minimal surface is determined in terms of $\tilde{\epsilon}$. In general, this area has a leading term that is proportional to the inverse of $\tilde{\epsilon}$. Subtracting this term and taking the suitable rescaling by the definition in (24), we can get the finite part of the minimal surface area that does not depend on $\tilde{\epsilon}$. We show our numerical results in various parameter regions in Fig. 2 and Fig. 3. In [53], the authors studied the system with momentum relaxation and showed their results in some parameter regions. Our results not only reproduce theirs well but also can be expanded to other parameter regions. Our result for the extended parameter space is telling us the role of impurity 


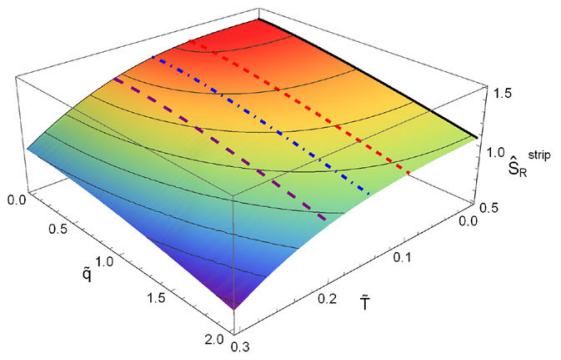

(a)

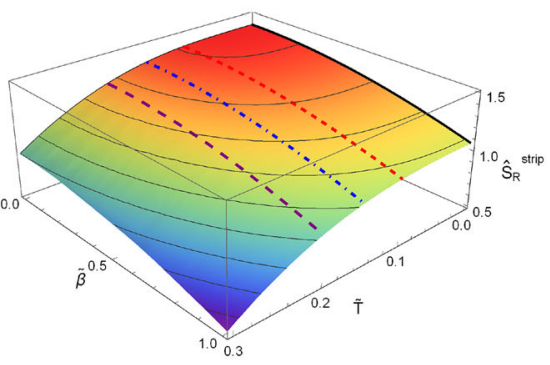

(d)

Fig. 2 The refined function $\hat{S}_{R}^{\text {strip }}$ at a $\tilde{\beta}=0, \mathbf{b} \tilde{\beta}=0.2$, c $\tilde{q}=0$, d $\tilde{q}=0.2$, e $\tilde{T}=0$ and $\mathbf{f} \tilde{T}=0.2:$ the lines on the surfaces denote $\tilde{T}$ $=0$ (Solid), 0.1 (Shortdashed), 0.15 (Dotdashed), 0.2 (Longdashed) and

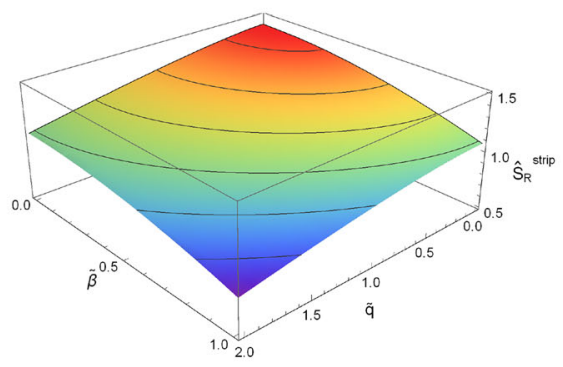

(e)

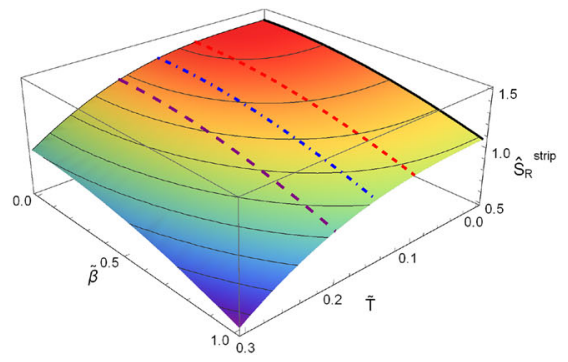

(c)

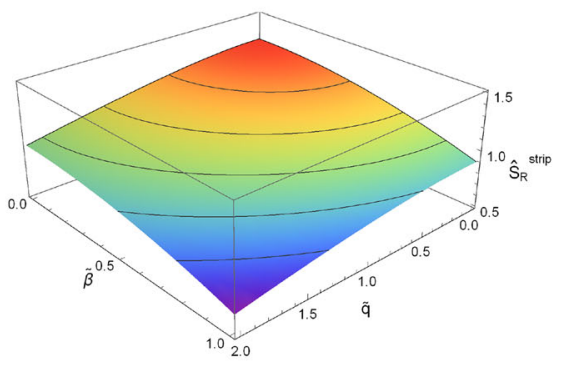

(f) these lines are plotted in Fig. 3. This result is easily extended to the entanglement entropy in the presence of magnetic field. Thus one may $\operatorname{regard} \tilde{q}$ and $\sqrt{\tilde{q}^{2}+\tilde{B}^{2}}$, where $\tilde{B}=F_{x y} l^{2}$

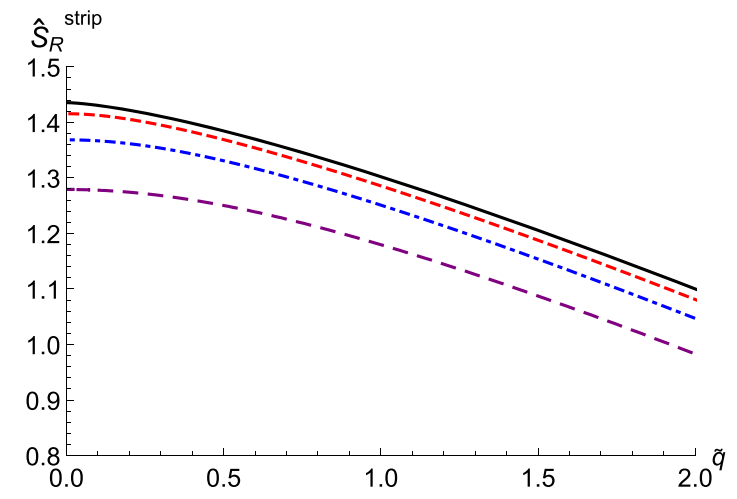

(a)

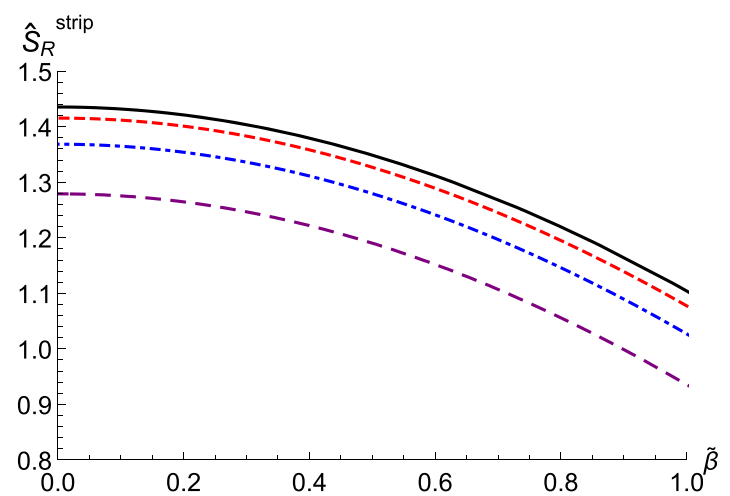

(c)

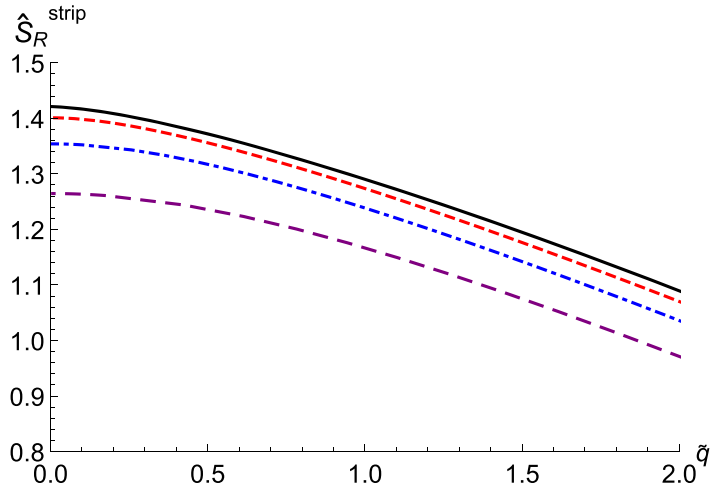

(b)

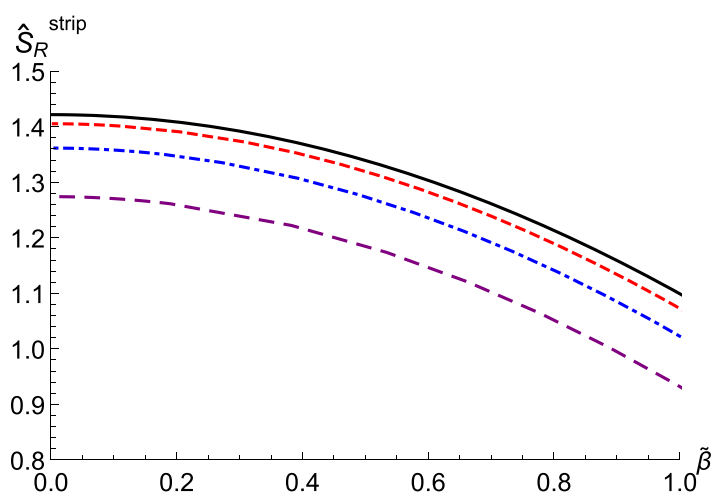

(d)

Fig. 3 The refined function $\hat{S}_{R}^{\text {strip }}$ for $\mathbf{a} \tilde{\beta}=0$, b $\tilde{\beta}=0.2$, $\mathbf{c} \tilde{q}=0$ and $\mathbf{d} \tilde{q}=0.2$. These curves represent the lines on the surfaces in Fig. 2 and correspond to the temperatures $\tilde{T}=0$ (Solid), 0.1 (Shortdashed), 0.15 (Dotdashed) and 0.2 (Longdashed) 
density, charge density and temperature in the entanglement entropy. As one can see, these parameters make the finite part of the entropy decrease. This finite part actually follows not an area law but a volume law [53].

It is noticeable that the above zeroth order calculation is invariant under the electromagnetic duality $(F \leftrightarrow \tilde{F})$ and also the rotation of $F$ and $\tilde{F}$ in the bulk. This property leads to the symmetry exchanging and rotating the charge density and the external magnetic field in the dual field theory [41, 59]. Thus the form of entanglement entropy can be extended to the entanglement entropy in the presence of the external magnetic field. The extended entanglement entropy is simply obtained by a transform: $\tilde{q}^{2} \rightarrow \tilde{q}^{2}+\tilde{B}^{2}$, where $\tilde{B}=B l^{2}$ and $B$ is nothing but the magnetic field $F_{x y}$ of the black brane. Therefore our result can cover the dyonic black brane case by a simple parameter change.

\subsection{Analytic result for holographic entanglement entropy}

Now we try to obtain analytic expression for the holographic entanglement entropy in the small $l$ limit. Our formulas (32) and (34) are also useful to study the analytic form. In order to study the analytic expression, one may take a small $\xi$ limit on both equations. This limit then allows us to integrate the above equations. We obtained the entanglement entropy in this limit as the following form :

$$
\begin{aligned}
S_{E E}^{\text {strip }} & =\frac{L}{4 G_{N} l}\left[\frac{2 l}{\epsilon}+\frac{\sqrt{2} \pi^{2} \Gamma\left(-\frac{1}{4}\right)}{\Gamma\left(\frac{1}{4}\right)^{3}}\right. \\
& +\frac{\pi r_{h}^{2} \gamma_{1} \Gamma\left(\frac{1}{4}\right)}{\sqrt{2} \Gamma\left(-\frac{1}{4}\right)^{2} \Gamma\left(\frac{7}{4}\right)} l^{2}+\frac{\pi r_{h}^{3}\left(2 \gamma_{1}-4-\gamma_{2}\right) \Gamma\left(\frac{1}{4}\right)}{\sqrt{2} \Gamma\left(-\frac{1}{4}\right)^{3}} l^{3} \\
& +\frac{r_{h}^{4}\left(576 \pi^{3}\left(3 \gamma_{1}^{2}-4 \gamma_{2}\right) \Gamma\left(\frac{3}{4}\right)-5 \sqrt{2} \gamma_{1}^{2} \Gamma\left(\frac{1}{4}\right)^{7}\right)}{92160 \pi^{2} \Gamma\left(\frac{3}{4}\right)^{5}} l^{4} \\
& +\frac{8 \pi^{2} r_{h}^{5} \gamma_{1}\left(2 \gamma_{1}-4-\gamma_{2}\right)\left(\pi^{3} \Gamma\left(-\frac{1}{4}\right)+48 \Gamma\left(\frac{3}{4}\right)^{5}\right)}{3 \Gamma\left(-\frac{1}{4}\right)^{7} \Gamma\left(\frac{3}{4}\right)^{6}} l^{5} \\
& \left.+\mathcal{O}\left(l^{6}\right)\right],
\end{aligned}
$$

where $r_{h}$ is given by $1 /\left(l z_{h}\right)$. In addition, the $\gamma_{1}$ and $\gamma_{2}$ are defined by

$\gamma_{1} \equiv \tilde{\beta}^{2} z_{h}^{2}, \quad \gamma_{2} \equiv \tilde{q}^{2} z_{h}^{4}$.

The physical meaning of this $\operatorname{limit}(\xi \ll 1)$ is that the tip distance $\left(z_{*}\right)$ from the boundary is much smaller than that of the horizon of the black hole $\left(z_{h}\right)$. Roughly speaking, this limit is similar to $\frac{l}{z_{h}} \ll 1$, but it is not the equivalent limit in a strict sense. The analytic result (35) is compared with the previous numerical calculations in Fig. 4.

In the present work, we considered the black hole involvling various parameters, temperature, charge density, and momentum relaxation. In general, the black hole horizon is given by a nontrivial function of these parameters. Our result indicates that the finite temperature effect reduces the entanglement entropy by washing out quantum entanglement entropy. In order to see the effects of the charge density and momentum relaxation, we think of their effect with a fixed black hole horizon. In this case, decreasing the charge density and momentum relaxation causes effectively the increase in temperature. This fact implies that similar to the increase in temperature, the decrease of the charge density and momentum relaxation gives the reduction of the entanglement entropy, see Fig. 3. Intuitively, the large momentum relaxation can easily occur in a high-density medium. Thus, we expect that the momentum relaxation gives rise to a similar effect to the density. In addition, as the density decreases, the reduction of the entanglement entropy can be understood as the following way. Noting that the entanglement entropy is linked to the degrees of freedom, we expect that the decrease of the density leads to the reduction of the degrees of freedom which may make quantum entanglement. This is another intuitive understanding for our results.

The above result can also have an extremal limit. At zero temperature, the charge density is given by $\tilde{q}=$ $\sqrt{12-2 z_{h}^{2} \tilde{\beta}} / z_{h}^{2}$. So the holographic entanglement entropy at zero temperature is determined to be:

$$
\begin{aligned}
S_{E E}^{\text {strip }} & =\frac{L}{4 G_{N} l}\left[\frac{2 l}{\epsilon}+\frac{\sqrt{2} \pi^{2} \Gamma\left(-\frac{1}{4}\right)}{\Gamma\left(\frac{1}{4}\right)^{3}}+\frac{64 \pi^{2} \beta^{2}}{3 \Gamma\left(-\frac{1}{4}\right)^{4}} l^{2}\right. \\
& +\frac{16 \pi^{2} r_{h}\left(4 r_{h}^{2}-\beta^{2}\right)}{\Gamma\left(-\frac{1}{4}\right)^{4}} l^{3} \\
& +\frac{8 \pi\left(-160 \pi^{2} \beta^{4} \Gamma\left(-\frac{3}{4}\right)^{2}+\left(3 \beta^{4}+8 r_{h}^{2} \beta^{2}-48 r_{h}^{4}\right) \Gamma\left(-\frac{1}{4}\right)^{6}\right)}{5 \Gamma\left(-\frac{1}{4}\right)^{10}} l^{4} \\
& \left.-\frac{8 \pi^{2} r_{h} \beta^{2}\left(4 r_{h}^{2}-\beta^{2}\right)\left(\pi^{3} \Gamma\left(-\frac{1}{4}\right)+48 \Gamma\left(\frac{3}{4}\right)^{5}\right)}{\Gamma\left(-\frac{1}{4}\right)^{7} \Gamma\left(\frac{3}{4}\right)^{5} \Gamma\left(\frac{7}{4}\right)} l^{5}+\mathcal{O}\left(l^{6}\right)\right] .
\end{aligned}
$$

This shows the effect of momentum relaxation on the holographic entanglement entropy when the temperature is zero.

The above two results quantitatively indicate contribution of impurity to the entanglement entropy. It is very interesting to compare these results with the effects of impurities in weakly coupled field theories. On the other hand, the leading contribution in (37) is proportional to $\beta^{2}$. It is remarkable that impurities reduce entanglement entropy in a very narrow strip and this result can be compared to known results in condensed matter physics, our system is different from systems studied in condensed matter physics though. See [60] and its references for recent articles. One may read [61] for a recent review.

As a final remark, we may think the strip case with a rotated electric field. This is achieved by replacing the subindex $x$ in (4) by the index $i$ denoting $(x, y)$ with nonvanishing $y$ - 


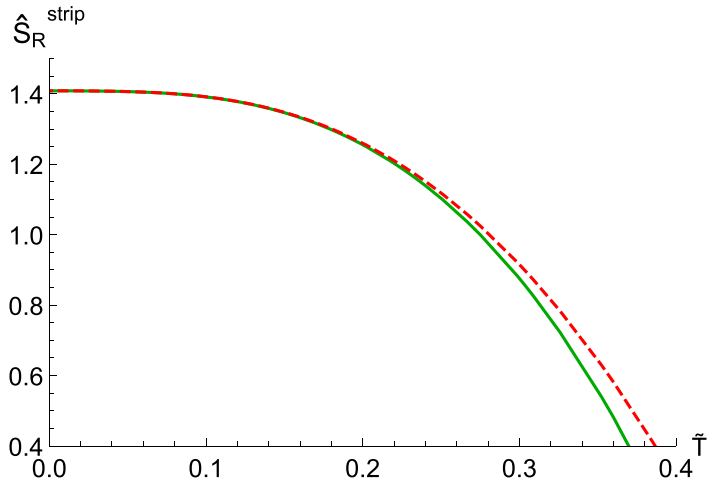

(a)

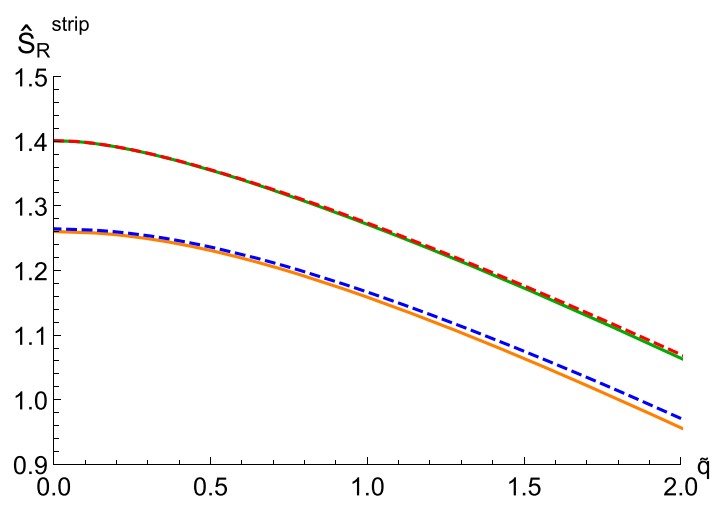

(c)

Fig. 4 Comparison of the numerical calculation(Dashed) and the analytic one(Solid) for $\tilde{\beta}=0.2$ : a is for a fixed charge density $\tilde{q}=0.2$ and $\mathbf{b}$ describes the difference, $\Delta$, between two results. As $\tilde{T}$ grows, $\Delta$ also increases. $\mathbf{c}$ Shows the case of a fixed temperature $\tilde{T}=0.1$ (Red-

components. However, the resulting action is still the same as the previous one up to the redefinition of the coordinate in 17 . This fact implies that the rotation of the electric field doesn't give any nontrivial contribution to the action of the minimal surface. In Sect. 4, we will consider the rotated electric field with a wedge entangling region which, even at the linear order, leads to a nontrivial effect on the entanglement entropy.

\subsection{A comparable deformation of ABJM theory}

Our results (35) and (37) tell us how the entanglement entropy changes under deforming the CFT vacuum with operators dual to the axion field and the bulk U(1) gauge field in (1). The background solution (2) describes the deformation more explicitly. Since we do not turn on any magnetic field, the operator dual to the gauge field is nothing but the charge density operator $\mathcal{Q}$ and the source is given by the chemical potential. In addition, the axion field which consists of two massless real scalar fields are dual to dimension 3 operators $\mathcal{O}_{I}^{\Delta=3}$.

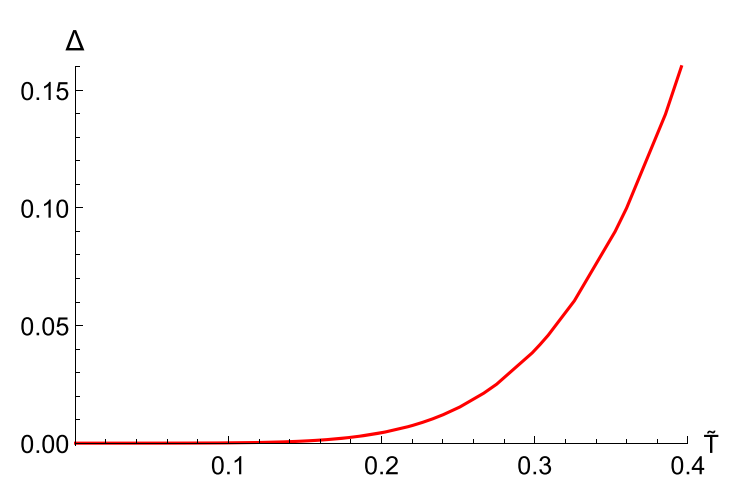

(b)

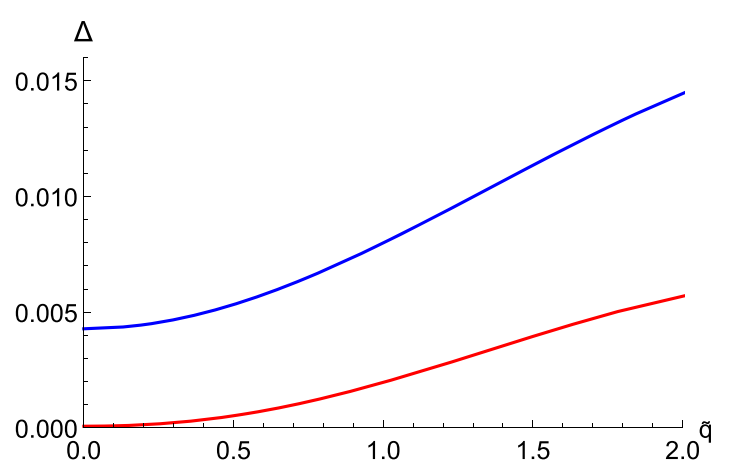

(d)

dashed, Green) and $\tilde{T}=0.2$ (Blue-dashed, Orange). In $\mathbf{d}, \Delta$ of the case $\tilde{T}=0.2$ (Blue) is larger than that of the case $\tilde{T}=0.1($ Red). When $\tilde{T}$ has a small value, the analytic result gives a good approximation

One of well-known examples of gauge/gravity correspondence is the duality between ABJM theory and $A d S_{4} \times S^{7} / \mathbb{Z}_{k}$ [52]. If we take the planar limit of ABJM theory, then the dual geometry becomes $A d S_{4} \times \mathbb{C P}_{3}$. Therefore the ABJM theory becomes dual to a 4-dimensional supergravity model that is obtained from the type IIA supergravity compactified on $\mathbb{C P}_{3}$. Thus one can find candidates for the fields in (1). The bulk local gauge symmetry appears as a global symmetry in the boundary. The ABJM theory has $S U$ (4) R-symmetry and the $U$ (1) symmetry can be embedded in the R-symmetry. So it is possible to identify $\mathcal{Q}$ with the charge operator of the diagonal $U(1)$ of $S U$ (4) R-symmetry, $\mathcal{Q}^{R}$. In addition there are many chiral primary scalar operators with dimension 3 , which are candidates for $\mathcal{O}_{I}^{\Delta=3}$. See [66] and table 1 in [67]. Therefore the following deformation of the ABJM theory is dual to (1):

$S^{\prime}=S_{A B J M}+\int d^{3} x\left(\mu \mathcal{Q}^{R}+\sum_{I=1}^{2} \mathcal{S}_{\Delta=3}^{I} \mathcal{O}_{I}^{\Delta=3}\right)$, 


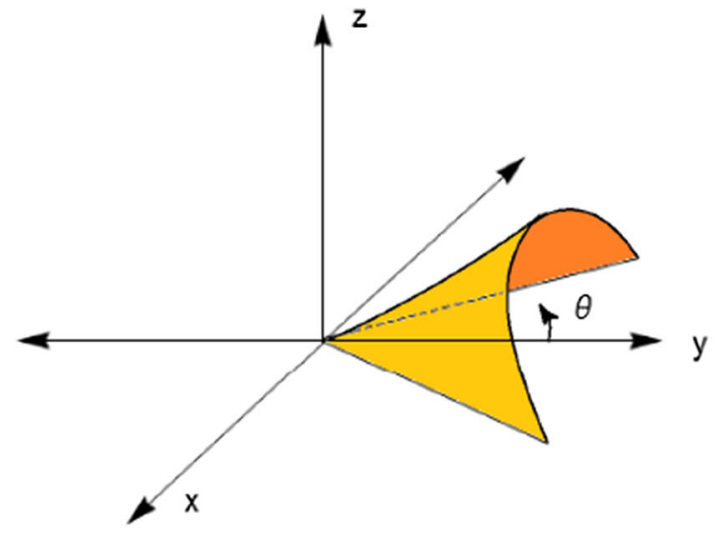

(a)

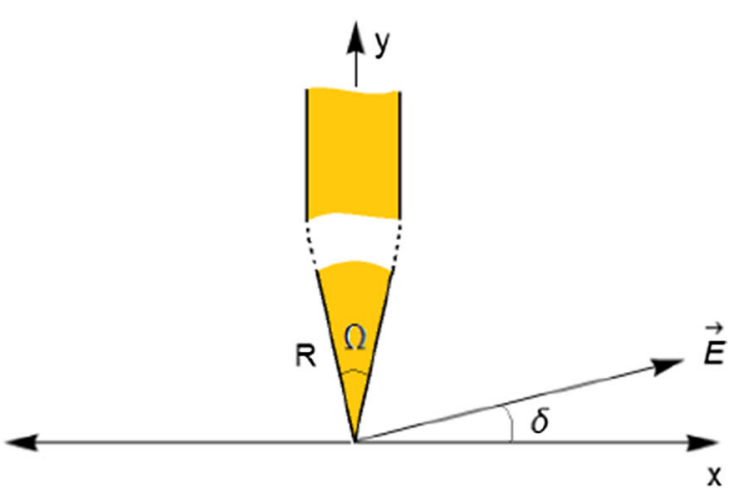

(b)

Fig. 5 Cartoons for a minimal surface (a) and its top view (b) : We regard the wedge type minimal surface as a part of a larger structure, such as a tail of a strip type minimal surface. We have depicted that imagination in the top view (b)

where $S_{A B J M}$ is the action of the ABJM theory and $\mathcal{S}_{\Delta=3}^{I}$ are source for the scalar operators in the ABJM theory. And it is given by $\mathcal{S}_{\Delta=3}^{I}=(\beta x, \beta y)$. There could be some difference coming from nonlinear effects of compactification on $\mathbb{C P}_{3}$. If, however, we consider small deformations and small size of the entangling region, the correction can be negligible. Then, it is valuable to compare the entanglement entropy with the above deformed action to (35) and (37). We hope to see a comparison of our result and the entanglement entropy obtained by a developing technique of supersymmetric field theory.

\section{Holographic entanglement entropy in electric field: wedge entangling region}

In this section, we consider the holographic entanglement entropy with wedge entangling regions. This type of entangling surfaces has been studied in $[16,17,68,69]$ to consider the corner contribution of entanglement entropy. It turns out that this corner contribution contains universal information of conformal field theory, which will be discussed later.

See Fig. 5 for a cartoon of a minimal surface anchored to a wedge region. It is useful to take polar coordinates $(\rho, \theta)$ in the $x-y$ plane as the coordinates of the minimal surface. Our main concern is how much the holographic entanglement entropy changes at the linear level under the weak electric field. To see this linear response, the direction of the electric field must be rotated by an angle $\delta$, and one of the Cartesian coordinates $x$ is given by $x=-\rho \sin (\theta-\delta)$. The need for the rotation was inferred by lessons of the previous section.

Considering the above configuration, the background geometry can be written as follows:

$$
\begin{aligned}
d s^{2}= & -U(r) d t^{2}+\frac{d r^{2}}{U(r)}+r^{2}\left(d \rho^{2}+\rho^{2} d \theta^{2}\right) \\
& -2 \lambda\left(g_{t x}(r) d t+g_{r x}(r) d r\right) \\
& \times(\rho \cos (\theta-\delta) d \theta+\sin (\theta-\delta) d \rho)+\mathcal{O}\left(\lambda^{2}\right) .
\end{aligned}
$$

And we may identify the coordinates of the minimal surface as the following way:

$t=0, \quad r=r\left(\sigma_{1}, \sigma_{2}\right)=1 / \tilde{z}\left(\sigma_{1}, \sigma_{2}\right), \quad \rho=\sigma_{1} . \quad \theta=\sigma_{2}$

Then the action for the minimal surface is given by

$$
\begin{aligned}
& \mathcal{A}=\int_{-\Omega / 2}^{\Omega / 2} d \theta \int_{0}^{\infty} d \rho \\
& \left(\frac{\rho}{\tilde{z}^{2}} \sqrt{1+\frac{\tilde{z}^{\prime 2}+\rho^{2} \dot{\tilde{z}}^{2}}{\rho^{2} \tilde{z}^{2} f(\tilde{z})}}+\lambda \frac{\mathbb{H}_{x x}\left(\tilde{z}^{\prime} \cos (\delta-\theta)-\rho \dot{\tilde{z}} \sin (\delta-\theta)\right)}{\tilde{z}^{2} f(\tilde{z}) \sqrt{1+\frac{\tilde{z}^{2}+\rho^{2} \dot{\tilde{z}}^{2}}{\rho^{2} \tilde{z}^{2} f(\tilde{z})}}}\right) .
\end{aligned}
$$

We are interested only in a variation of the action under the electric field and regard the wedge as a part of a larger minimal surface like the cartoon (b) in Fig. 5. So we consider a part of the minimal surface with the region, $\rho<R$ and we will see how the surface changes for this finite region. By this reason, the integration for the radial coordinate $\rho$ is replaced by the integration between 0 to $R$. Furthermore, we will take a convenient choice of parameters obtained by a scaling with respect to $\Omega$ :

$$
\begin{aligned}
\sigma=\theta / \Omega, & \tilde{z}=\Omega w, \quad \tilde{\beta}=\Omega \beta, \\
\tilde{M}=\Omega^{3} M & \tilde{q}=\Omega^{2} q .
\end{aligned}
$$


Then, the action becomes

$$
\begin{aligned}
\mathcal{A}= & \frac{1}{\Omega} \int_{-1 / 2}^{1 / 2} d \sigma \int_{0}^{R} d \rho\left(\frac{\rho}{w^{2}} \sqrt{1+\frac{w^{\prime 2}+\Omega^{2} \rho^{2} \dot{w}^{2}}{\rho^{2} w^{2} f(w)}}\right. \\
& \left.+\lambda \Omega^{2} \frac{\mathbb{H}_{r x}\left(w^{\prime} \cos (\delta-\Omega \sigma)-\Omega \rho \dot{w} \sin (\delta-\Omega \sigma)\right)}{w^{2} f(w) \sqrt{1+\frac{w^{\prime 2}+\Omega^{2} \rho^{2} \dot{w}^{2}}{\rho^{2} w^{2} f(w)}}}\right),
\end{aligned}
$$

where $f(w)$ is given by

$f(w)=\frac{1}{w^{2}}-\frac{\tilde{\beta}^{2}}{2}-\tilde{M} w+\frac{\tilde{q}^{2}}{4} w^{2}$.

The above action determines the minimal surface by solving the equation of motion for $w(\rho, \sigma)$. In general, the equation is not easy to solve.

Now let us take a particular limit considering a sharp wedge. Then one can use $\Omega$ as another small parameter. The action up to the quadratic order in $\Omega$ is given by

$$
\begin{aligned}
\mathcal{A}= & \frac{1}{\Omega} \int_{-1 / 2}^{1 / 2} d \sigma \int_{0}^{R} d \rho \\
& \times\left(\sum_{n=0}^{3} \mathcal{L}_{(n)}^{0 t h} \Omega^{n}+\lambda \sum_{n=2}^{3} \mathcal{L}_{(n)}^{1 s t} \Omega^{n}+\mathcal{O}\left(\Omega^{4}\right)\right),
\end{aligned}
$$

where

$$
\begin{aligned}
& \mathcal{L}_{(0)}^{0 t h}=\frac{\rho \sqrt{\frac{w^{\prime 2}}{\rho^{2}}+1}}{w^{2}}, \quad \mathcal{L}_{(1)}^{0 t h}=0, \\
& \mathcal{L}_{(2)}^{0 t h}=\frac{\left(\beta^{2} w^{2}\left(w^{\prime}\right)^{2}+2 \rho^{2} \dot{w}^{2}\right)}{4 \rho w^{2} \sqrt{\frac{\left(w^{\prime}\right)^{2}}{\rho^{2}}+1}}, \\
& \mathcal{L}_{(3)}^{0 t h}=\frac{M w w^{\prime 2}}{2 \rho \sqrt{\frac{w^{\prime 2}}{\rho^{2}}+1}}, \\
& \mathcal{L}_{(2)}^{1 s t}=\mathbb{H}_{r x} \frac{w^{\prime} \cos \delta}{\sqrt{\frac{w^{\prime 2}}{\rho^{2}}+1}} \text { and } \\
& \mathcal{L}_{(3)}^{1 s t}=\mathbb{H}_{r x} \frac{\left(\sigma w^{\prime}-\rho \dot{w}\right) \sin \delta}{\sqrt{\frac{w^{\prime 2}}{\rho^{2}}}+1} .
\end{aligned}
$$

Since $\Omega$ is considered small, the solution $w$ is closed to the form of $\rho$ times a function of $\sigma$. Thus we assume that $w$ can be represented by a polynomial of $\rho$. Under this assumption, we found a solution of the equation of motion as the following form:

$$
\begin{aligned}
w(\rho, \sigma) & =w_{0}(\rho, \sigma)+\lambda w_{1}(\rho, \sigma) \\
& =\rho h_{0,1}(\sigma)+\Omega^{2}\left(\sum_{q=1}^{3} h_{2, q}(\sigma) \rho^{q}\right)+\Omega^{3} h_{3,4}(\sigma) \rho^{4}
\end{aligned}
$$

$$
+\lambda \mathbb{H}_{r x}\left(\Omega^{2} g_{2,3}(\sigma) \rho^{3} \cos \delta+\Omega^{3} g_{3,3}(\sigma) \rho^{3} \sin \delta\right)
$$

The differential equations for the functions, $h_{p, q}$ and $g_{p, q}$ are given in Appendix A. In addition, the regularized area is given by

$$
\begin{aligned}
\mathcal{A}_{\text {reg }}= & \frac{1}{\Omega} \int_{\sigma_{\min }}^{\sigma_{\max }} d \sigma \int_{\alpha(\sigma)+\lambda \beta(\sigma)}^{R} d \rho \\
& \times\left(\sum_{n=0}^{3} \mathcal{L}_{(n)}^{0 t h} \Omega^{n}+\lambda \sum_{n=2}^{3} \mathcal{L}_{(n)}^{1 s t} \Omega^{n}\right),
\end{aligned}
$$

where $\alpha(\sigma)+\lambda \beta(\sigma)$ is the cut-off line obtained from $\tilde{z}=\epsilon$ and the explicit form is given in Appendix A. In this regard, $\sigma_{\max }$ and $\sigma_{\min }$ denote the maximum and minimum values of $\sigma$ satisfying $\alpha(\sigma)+\lambda \beta(\sigma)=R$. And they can be expanded as $\sigma_{\text {max } \text { min }} \sim \sigma_{\text {max } \text { min }}^{(0)}+\lambda \sigma_{\text {max } \text { min }}^{(1)}$, respectively. The regularised action to the linear order in $\lambda$ is

$$
\begin{aligned}
\mathcal{A}_{\text {reg }}= & \frac{1}{\Omega} \int_{\sigma_{\min }^{(0)}}^{\sigma_{\max }^{(0)}} d \sigma \int_{\alpha(\sigma)}^{R} d \rho \\
& \times\left[\left(\sum_{n=0}^{3} \mathcal{L}_{(n)}^{0 t h} \Omega^{n}\right)_{w_{0}+\lambda w_{1}}+\lambda\left(\sum_{n=2}^{3} \mathcal{L}_{(n)}^{1 s t} \Omega^{n}\right)_{w_{0}}\right] \\
& +\frac{1}{\Omega}\left(\int_{\sigma_{\min }}^{\sigma_{\max }} d \sigma \int_{\alpha(\sigma)+\lambda \beta(\sigma)}^{R} d \rho\right. \\
& \left.-\int_{\sigma_{\min }^{(0)}}^{\sigma_{\max }^{(0)}} d \sigma \int_{\alpha(\sigma)}^{R} d \rho\right)\left(\sum_{n=0}^{3} \mathcal{L}_{(n)}^{0 t h} \Omega^{n}\right)_{w_{0}}+\mathcal{O}\left(\lambda^{2}\right) .
\end{aligned}
$$

Plugging (48) into the above action, we can find the onshell action as follows:

$$
\begin{aligned}
\mathcal{A}_{r e g} \sim & \frac{1}{\Omega} \int_{\sigma_{\min }^{(0)}}^{\sigma_{\max }^{(0)}} d \sigma \int_{\alpha(\sigma)}^{R} d \rho\left(\sum_{n=0}^{3} \mathcal{L}_{(n)}^{0 t h} \Omega^{n}\right)_{w_{0}} \\
& -\frac{\lambda \mathbb{H}_{r x}}{\Omega} \cos \delta \int_{-\sigma_{\max }^{(0)}}^{\sigma_{\max }^{(0)}} d \sigma \\
& \times \int_{\alpha(\sigma)}^{R} d \rho \frac{\rho \Omega^{2}\left(2 g_{2,3}\left(h_{0,1}^{\prime 2}+1\right)-h_{0,1} g_{2,3}^{\prime} h_{0,1}^{\prime}\right)}{h_{0,1}^{3} \sqrt{h_{0,1}^{\prime 2}+1}} \\
& -\frac{\lambda \mathbb{H}_{r x}}{\Omega} \sin \delta \int_{-\sigma_{\max }^{(0)}}^{\sigma_{\max }^{(0)}} d \sigma \\
& \times \int_{\alpha(\sigma)}^{R} d \rho \frac{\rho \Omega^{3}\left(2 g_{3,3}\left(h_{0,1}^{\prime 2}+1\right)-h_{0,1} g_{3,3}^{\prime} h_{0,1}^{\prime}\right)}{h_{0,1}^{3} \sqrt{h_{0,1}^{\prime 2}+1}} \\
& +\frac{\lambda \mathbb{H}_{r x}}{\Omega} \int_{-\sigma_{\max }^{(0)}}^{\sigma_{\max }^{(0)}} d \sigma \\
& \times \int_{\alpha(\sigma)}^{R} d \rho \rho\left(\frac{\Omega^{2} h_{0,1}^{\prime} \cos \delta}{\sqrt{h_{0,1}^{\prime 2}+1}}+\frac{\Omega^{3}\left(\sigma h_{0,1}^{\prime}-h_{0,1}\right) \sin \delta}{\sqrt{h_{0,1}^{\prime 2}+1}}\right)
\end{aligned}
$$




$$
\begin{aligned}
& +\frac{\lambda}{\Omega}\left(\sigma_{\max }^{(1)}-\sigma_{\min }^{(1)}\right)\left(\int_{\alpha(\sigma)}^{R} d \rho\left(\sum_{n=0}^{3} \mathcal{L}_{(n)}^{0 t h} \Omega^{n}\right)_{w_{0}}\right)_{\sigma=\sigma_{\max }^{(0)}} \\
& -\frac{\lambda}{\Omega} \int_{-\sigma_{\max }^{(0)}}^{\sigma_{\max }^{(0)}} d \sigma \beta(\sigma)\left(\sum_{n=0}^{3} \mathcal{L}_{(n)}^{0 t h} \Omega^{n}\right)_{\rho=\alpha(\sigma)}
\end{aligned}
$$

where we used $\sigma_{\text {min }}^{(0)}=-\sigma_{\text {max }}^{(0)}$. And the first order correction for $\sigma_{\min }$ and $\sigma_{\max }$ is given by

$\sigma_{\min , \max }^{(1)}=-\frac{\beta\left(\sigma_{\min , \max }^{(0)}\right)}{\alpha^{\prime}\left(\sigma_{\min , \max }^{(0)}\right)}$.

This can be easily derived from $R=\alpha\left(\sigma_{\min , \max }\right)+$ $\lambda \beta\left(\sigma_{\min , \max }\right)$.

The zeroth order part in $\lambda$ is given by the first line in (51). In fact this part shows universal features of underlying CFTs $[16,17,70,71]$, which are summarized as follows. This term has two kinds of divergent terms. One is the usual UV divergent term which is proportional to $1 / \epsilon$ that appears commonly in $2+1$ dimensional field theory systems. One can see the term also in the strip case, (35). In addition to this, another log-divergent term shows up. Since this term is coming from the singular corner of the wedge, it is called the corner contribution. The general structure of the holographic entanglement entropy is as follows:

$S_{E E}^{(0)}=\frac{1}{4 G_{N}} \mathcal{A}_{r e g}^{(0)}=\mathbb{B} \frac{\mathbb{L}}{\epsilon}-\mathbb{A}(\Omega) \log \frac{\mathbb{L}}{\epsilon}+\mathbb{C}^{(0)}+\mathcal{O}\left(\frac{\epsilon}{\mathbb{L}}\right)$,

where $\mathbb{L}$ is a typical length scale characterized by the size of the entangling region. Even though the constant $\mathbb{B}$ governs the leading term, it crucially depends on UV regulators. $\mathbb{C}^{(0)}$ is the finite part of entanglement entropy. The most interesting quantity is the coefficient of log-term, $\mathbb{A}(\Omega)$. The characteristic features of $\mathbb{A}$ are determined by two limits of $\Omega$ :

$\lim _{\Omega \rightarrow 0} \mathbb{A} \sim \frac{\kappa_{1}}{\Omega}, \quad \lim _{\Omega \rightarrow \pi} \mathbb{A} \sim \sigma_{1}(\pi-\Omega)^{2}$,

where $\kappa_{1}$ is conjectured to be related to, so called, entropic c-function and $\sigma_{1}$ is given by $\sigma_{1}=\frac{\pi^{2}}{24} C_{T}$. $C_{T}$ is the central charge appearing in the vacuum two point function as follows:

$\left\langle T_{\mu \nu}(x) T_{\alpha \beta}(0)\right\rangle=\frac{C_{T}}{|x|^{4}} \mathcal{I}_{\mu \nu, \alpha \beta}(x)$

where $\mathcal{I}_{\mu \nu, \alpha \beta}$ is a dimesionless tensor which is completely fixed by conformal symmetry [72]. It is worth noting that the coefficient of the log-divergent term $\mathbb{A}$ is UV regulator independent, as opposed to $\mathbb{B}$. In addition these results were generalized to the Reyni entropy. See [17] and references therein.
Even though the above zeroth order part is very interesting, our main interest here is the linear order variation. So we focus on the first order part. If we write down only the first order part using some algebra in Appendix B, the linear action in $\lambda$ is :

$\delta \mathcal{A}_{\text {reg }}=-\lambda \mathbb{H}_{r x} \Omega^{2} \sin \delta \int_{-\sigma_{\max }^{(0)}}^{\sigma_{\max }^{(0)}} d \sigma\left(\mathcal{I}_{1}+\mathcal{I}_{2}\right)+\mathcal{O}\left(\Omega^{3}\right)$,

where

$\mathcal{I}_{1}=\frac{\left(R^{2}-\alpha(\sigma)^{2}\right)\left(2 g_{3,3}(\sigma)\left(h_{0,1}^{\prime}(\sigma)^{2}+1\right)-h_{0,1}(\sigma) g_{3,3}^{\prime}(\sigma) h_{0,1}^{\prime}(\sigma)\right)}{2 h_{0,1}(\sigma)^{3} \sqrt{h_{0,1}^{\prime}(\sigma)^{2}+1}}$,

$\mathcal{I}_{2}=\left(R^{2}-\alpha(\sigma)^{2}\right)\left(-\frac{\lambda\left(\sigma h_{0,1}^{\prime}(\sigma)-h_{0,1}(\sigma)\right)}{2 \sqrt{h_{0,1}^{\prime}(\sigma)^{2}+1}}\right)$.

Here the contribution of $\alpha(\sigma)^{2}$ term to the integration is much smaller than the contribution of $R^{2}$ term. See Appendix B for the explicit form of $\alpha(\sigma)$ that is order of $\mathcal{E}$. Thus we drop the $\alpha(\sigma)^{2}$ term.

If we drop out the discussed higher orders in $\epsilon$ and the integrations of some odd functions, ${ }^{5}$ the leading change of the regularized minimal surface to the external electric field is given by

$$
\begin{aligned}
& \delta \mathcal{A}_{r e g}=\lambda R^{2} \Omega^{2} \mathbb{H}_{r x} \sin \delta \int_{-\sigma_{\max }^{(0)}}^{\sigma_{\max }^{(0)}} d \sigma \\
& \times \frac{-\sigma h(\sigma)^{3} h^{\prime}(\sigma)+h(\sigma)^{4}+h(\sigma) h^{\prime}(\sigma) g^{\prime}(\sigma)-2 g(\sigma)\left(h^{\prime}(\sigma)^{2}+1\right)}{2 h(\sigma)^{3} \sqrt{h^{\prime}(\sigma)^{2}+1}},
\end{aligned}
$$

where $h(\sigma)=h_{0,1}(\sigma)$ and $g(\sigma)=-g_{3,3}(\sigma)$. In addition $g$ and $h$ satisfy the following equations :

$\begin{aligned} h^{\prime \prime} & =-\frac{2\left(h^{\prime 2}+1\right)}{h}, \\ g^{\prime \prime} & =-\frac{4 g^{\prime} h^{\prime}}{h}+\frac{2 g\left(1+h^{\prime 2}\right)}{h^{2}}-2 h^{2}+6 \sigma h h^{\prime}+4 h^{2} h^{\prime 2} .\end{aligned}$

One can solve the above equations numerically and perform the numerical integration in (59). Finally, we can get the linear response of the holographic entanglement entropy to the electric field as follows.

$\frac{\delta \mathcal{S}_{E E}}{\delta E_{x}}=\frac{R^{2} \Omega^{2}}{4 G_{N}} \mathcal{N} \frac{\bar{\alpha}}{4 \pi} \sin \delta$,

\footnotetext{
$\overline{5}$ One can easily see that all $h_{p, q}$ 's and $g_{3,3}$ are even functions and $g_{2,3}$ is an odd function from the equations in Appendix A.
} 
where $\mathcal{N}$ is the integration in (59). The integrand of $\mathcal{N}$ is a positive function over $-\frac{1}{2} \leq \sigma \leq \frac{1}{2}$, thus $\mathcal{N}$ does not vanish and it is about 0.05 . Therefore, the linear response of the holographic entanglement entropy to the electric field is proportional to the thermoelectric conductivity $\bar{\alpha}$ which is a measurable quantity.

Intriguingly, the above result shows a novel relationship between the entanglement entropy and linear response. On the contrary to the present work, the authors in $[49,62]$ investigated the evolution of a quenched system with U(1) current by using the method developed in $[63,64]$. They showed that in the quenched system, the first law of entanglement entropy satisfies the relation, $\partial_{t} \delta S_{E E} \propto \sigma E^{2}$. In [49], the authors focus on the fluctuation of a small mass parameter, $m \propto E^{2}$, which is determined by the regularity condition at the horizon with an electric field [65] However, we showed in the present work that the entanglement entropy of a rotated wedge entangling region can lead to $\delta S_{E E} \propto \bar{\alpha} E \sin \delta$. The reason we obtained the entanglement entropy correction proportional to $E$ is that we took into account the rotation (denoted by $\delta$ ) of the wedge entangling region. For $\delta=0$, this linear term disappears and the next term proportional to $E^{2}$ occurs as a leading correction, which is consistent with the result of $[49,62]$. In condensed matter community, related physics is a quite active research topic and has produced many interesting results. See [61] for a review. The origin of the linearity of the thermal conductivity $\bar{\alpha}$ comes from turning on the metric component $g_{r x}$ by the external electric field. Together with a reasonable gauge choice, this component describes the thermoelectric current. This hallmark is universal in various holographic models with momentum relaxation. Therefore, the above linear relation between the entanglement entropy variation and the thermoelectric coefficient is quite noticeable as a universal feature of entanglement in strongly coupled systems. Naturally, one can ask the reason why the leading effect is from the heat current mediated by the thermoelectric coefficient not from the electric current by the electric conductivity. This question is very difficult to answer because such an effect is closely related to the Fermi surface. We leave this investigation for our future work.

The measurement of entanglement is a very intriguing topic in various areas of condensed matter physics. The first measurement of the second Rényi entropy was accomplished in [73]. This work was inspired by [74,75]. Except for this measurement, there are various trials to detect entanglement measure. Although the accomplished detections were seen through few-body correlations, there are many discussions and proposals on extension of the experiments. Among them, a suitable proposal to our result is based on quantum antiferromagnets. See [77]. In the proposal, the entanglement measure can be encoded in magnetization of a system. A circular superconducting plate covers on both sides of an antiferromagnet sheet. By the Meissner effect, the plate plays a role of dividing entangling regions. Assuming that such an experiment is realized, we propose an experiment with a wedge type superconducting plate and applying an rotated electric field along a tangential direction. Then, one may check whether resulting entanglement entropy data is related to the thermoelectric coefficient $\bar{\alpha}$ or not. Our idea is a bit rough, but worth thinking about more.

\section{Discussion}

In this work, we have studied the entanglement entropy affected by the external electric field. Though the entanglement entropy has usually been considered as an important and fundamental quantity representing a variety of quantum natures, it still remains a big issue to measure the quantum entanglement entropy in the laboratory. One of the main goals of this work is how we can relate the quantum entanglement entropy to the measurable quantities like the transport coefficients. This would be useful to figure out the relation between the quantum phenomena and various macroscopic quantities. Moreover, the present work may provide new intuitions about how to measure the entanglement entropy in the laboratory.

More specifically, we have taken into account the entanglement entropy of the strip and wedge entangling region with turning on the electric field and momentum relaxation. If there is no momentum relaxation which breaks the translation symmetry, the DC conductivity usually has an infinite value. In order to obtain a finite conductivity in the zero frequency limit, we first considered the geometry with the non-vanishing momentum relaxation which resembles introducing impurities to the dual field theory. This momentum relaxation usually modifies the electric and thermoelectric conductivities. Theoretically, those changes of the transport coefficients can be governed by the response theory and we can easily measure such changes experimentally in the laboratory. In this situation, we can ask how the entanglement entropy is affected by the external electric field and what is the relation between the modified entanglement entropy and transport coefficients. In this paper, we showed how the entanglement entropy modified by the external electric field using the holographic method. It turns out that the entanglement entropy change can be connected to the transport coefficient, especially the thermoelectric conductivity.

For the strip case, we found that turning on the external electric field tilted the minimal surface as expected. In the holographic model, the area of the minimal surface is directly associated with the entanglement entropy. Thus, we may expect the change of the entanglement entropy due to the external electric field. We found that such a change of the entanglement entropy does not occur at least in the linear response theory. This is because the holographic formula of the entanglement entropy in (18) has an invariant form under 
the parity transformation like $x \rightarrow-x$. Despite the tilted minimal surface, the invariance under the parity transformation does not allow the change of the entanglement entropy at least at the linear order. However, the higher order corrections can affect the resulting entanglement entropy. In fact, we explicitly showed that the second order correction caused by the tilted minimal surface can change the entanglement entropy, although we did not regard an additional contribution caused by the background metric deformation. However, we did not present the result in this note because we leave it as part of future works. The additional contribution is related to the response theory at the second order and we hope to report more results on this issue in future works. Anyway, the results in the strip entangling region showed the fact that the change of the entanglement entropy can be represented in terms of transport coefficients.

In order to get more clear and explicit relation at the linear order, we further considered the entanglement entropy in the wedge entangling region which is utilized to extract universal information about the corner contribution. As shown in the strip case, the entanglement entropy invariant under the parity transformation does not give any nontrivial contribution at the linear order, so that we took into account the external electric field which is rotated with an arbitrary angle. Since the external electric field with an arbitrary rotation usually breaks the parity invariance, one can expect the nontrivial contribution to the entanglement entropy even at the linear order. We showed with explicit calculations that the linear order correction to entanglement entropy really occurs in this setup. Intriguingly, we furthermore found that the change of the entanglement entropy is directly related to the thermoelectric conductivity. This is the first example showing how the entanglement entropy can be connected to the transport coefficients. In addition we provided an rough experimental idea for our result of the wedge case and we also described a comparable deformation in the ABJM theory to the strip case. In future works, we hope to report more evidences and understanding of the underlying structure of the connection between the entanglement entropy and transport coefficients.

Acknowledgements K. Kim appreciates APCTP for its hospitality during completion of this work. This work was supported by the Korea Ministry of Education, Science and Technology, GyeongsangbukDo and Pohang City. C. Park was also supported by Basic Science Research Program through the National Research Foundation of Korea funded by the Ministry of Education (NRF-2016R1D1A1B03932371). J. Lee was supported by the same fund with grant number NRF2018R1A6A3A11049655. K. Kim and B. Ahn were supported by the same fund with grant number NRF-2015R1D1A1A01058220. K. Kim was also supported by the same fund with grant number NRF2019R1A2C1007396. This research was partially (B. Ahn) supported by the National Research Foundation of Korea(NRF) grant with the grant number NRF-2016R1D1A1A09917598, by the Yonsei University Future-leading Research Initiative of 2017(2017-22-0098) and by the Graduate School of YONSEI University Research Scholarship Grants in 2000 .
Data Availability Statement This manuscript has associated data in a data repository. [Authors' comment: All analyzed data have been published by the respective collaborations and are available online on HEPData.]

Open Access This article is distributed under the terms of the Creative Commons Attribution 4.0 International License (http://creativecomm ons.org/licenses/by/4.0/), which permits unrestricted use, distribution, and reproduction in any medium, provided you give appropriate credit to the original author(s) and the source, provide a link to the Creative Commons license, and indicate if changes were made.

Funded by SCOAP . $^{3}$

\section{Appendix}

\section{A. Equations for $h_{p, q}$ and $g_{p, q}$}

$h_{0,1}^{\prime \prime}=-\frac{2\left(\left(h_{0,1}^{\prime}\right)^{2}+1\right)}{h_{0,1}}$
$h_{2,1}^{\prime \prime}=\frac{h_{0,1}^{3}\left(2\left(h_{0,1}^{\prime}\right)^{2}-1\right)-4 h_{0,1} h_{0,1}^{\prime} h_{2,1}^{\prime}+2 h_{2,1}\left(\left(h_{0,1}^{\prime}\right)^{2}+1\right)}{h_{0,1}^{2}}$

$h_{2,2}^{\prime \prime}=\frac{2\left(h_{2,2}\left(\left(h_{0,1}^{\prime}\right)^{2}+1\right)-2 h_{0,1} h_{0,1}^{\prime} h_{2,2}^{\prime}\right)}{h_{0,1}^{2}}$

$h_{2,3}^{\prime \prime}=-\frac{\beta^{2} h_{0,1}^{3}\left(\left(h_{0,1}^{\prime}\right)^{2}-2\right)+8 h_{0,1} h_{0,1}^{\prime} h_{2,3}^{\prime}-4 h_{2,3}\left(\left(h_{0,1}^{\prime}\right)^{2}+1\right)}{2 h_{0,1}^{2}}$

$h_{3,4}^{\prime \prime}=\frac{M h_{0,1}^{4}\left(4-3\left(h_{0,1}^{\prime}\right)^{2}\right)-8 h_{0,1} h_{0,1}^{\prime} h_{3,4}^{\prime}+4 h_{3,4}\left(\left(h_{0,1}^{\prime}\right)^{2}+1\right)}{2 h_{0,1}^{2}}$

$g_{2,3}^{\prime \prime}=-\frac{4 g_{2,3}^{\prime} h_{0,1}^{\prime}}{h_{0,1}}+\frac{2 g_{2,3}\left(\left(h_{0,1}^{\prime}\right)^{2}+1\right)}{h_{0,1}^{2}}-6 h_{0,1} h_{0,1}^{\prime}$

$$
\begin{aligned}
g_{3,3}^{\prime \prime}= & -\frac{4 g_{3,3}^{\prime} h_{0,1}^{\prime}}{h_{0,1}}+\frac{2 g_{3,3}\left(\left(h_{0,1}^{\prime}\right)^{2}+1\right)}{h_{0,1}^{2}} \\
& +\frac{2\left(-3 \sigma h_{0,1}^{3} h_{0,1}^{\prime}-2 h_{0,1}^{4}\left(h_{0,1}^{\prime}\right)^{2}+h_{0,1}^{4}\right)}{h_{0,1}^{2}} \\
\alpha(\sigma)= & \frac{\mathcal{E}}{h_{0,1}}+\Omega^{2}\left(-\frac{\mathcal{E}^{3} h_{2,3}}{h_{0,1}^{4}}-\frac{\mathcal{E}^{2} h_{2,2}}{h_{0,1}^{3}}-\frac{\mathcal{E} h_{2,1}}{h_{0,1}^{2}}\right) \\
& -\Omega^{3} \frac{\mathcal{E}^{4} h_{3,4}}{h_{0,1}^{5}}
\end{aligned}
$$


$\beta(\sigma)=-\frac{\mathcal{E}^{3} \Omega^{2} g_{2,3} \mathbb{H}_{\mathrm{rx}}}{h_{0,1}^{4}} \sin \delta-\frac{\mathcal{E}^{3} \Omega^{3} g_{3,3} \mathbb{H}_{\mathrm{rx}}}{h_{0,1}^{4}} \cos \delta$,

where $\mathcal{E}=\frac{\epsilon}{\Omega}$ is a scaled cut-off.

\section{B. Detailed Calculation for (22)}

Since $\alpha(\sigma)$ and $h_{0,1}$ are even functions and $g_{2,3}$ is an odd function,

$$
\begin{aligned}
& -\frac{\lambda \mathbb{H}_{r x}}{\Omega} \cos \delta \\
& \quad \times \int_{-\sigma_{\max }^{(0)}}^{\sigma_{\max }^{(0)}} d \sigma \int_{\alpha(\sigma)}^{R} d \rho \frac{\rho \Omega^{2}\left(2 g_{2,3}\left(h_{0,1}^{\prime 2}+1\right)-h_{0,1} g_{2,3}^{\prime} h_{0,1}^{\prime}\right)}{h_{0,1}^{3} \sqrt{h_{0,1}^{\prime 2}+1}}=0 .
\end{aligned}
$$

By the same reason,

$$
\begin{aligned}
& \frac{\lambda \mathbb{H}_{r x}}{\Omega} \\
& \quad \times \int_{-\sigma_{\max }^{(0)}}^{\sigma_{\max }^{(0)}} d \sigma \int_{\alpha(\sigma)}^{R} d \rho \rho\left(\frac{\Omega^{2} h_{0,1}^{\prime} \cos \delta}{\sqrt{h_{0,1}^{\prime 2}+1}}+\frac{\Omega^{3}\left(\sigma h_{0,1}^{\prime}-h_{0,1}\right) \sin \delta}{\sqrt{h_{0,1}^{\prime 2}+1}}\right) \\
& =\frac{\lambda \mathbb{H}_{r x}}{\Omega} \sin \delta \times \int_{-\sigma_{\max }^{(0)}}^{\sigma_{\max }^{(0)}} d \sigma \int_{\alpha(\sigma)}^{R} d \rho \rho\left(\frac{\Omega^{3}\left(\sigma h_{0,1}^{\prime}-h_{0,1}\right)}{\sqrt{h_{0,1}^{\prime 2}+1}}\right) .
\end{aligned}
$$

In addition,

$$
\begin{aligned}
& \frac{\lambda}{\Omega}\left(\sigma_{\max }^{(1)}-\sigma_{\min }^{(1)}\right)\left(\int_{\alpha(\sigma)}^{R} d \rho\left(\sum_{n=0}^{3} \mathcal{L}_{(n)}^{0 t h} \Omega^{n}\right)_{w_{0}}\right)_{\sigma=\sigma_{\max }^{(0)}} \\
& =\frac{\lambda}{\Omega}\left(-\frac{\beta\left(-\sigma^{0}{ }_{\max }\right)+\beta\left(\sigma^{0}{ }_{\max }\right)}{\alpha^{\prime}\left(\sigma^{0}{ }_{\max }\right)}\right) \\
& \times\left(\int_{\alpha(\sigma)}^{R} d \rho\left(\sum_{n=0}^{3} \mathcal{L}_{(n)}^{0 t h} \Omega^{n}\right)_{w_{0}}\right)_{\sigma=\sigma_{\max }^{(0)}} \\
& =\frac{\lambda}{\Omega}\left(-\frac{2 g_{3,3} \mathcal{E}^{2}}{h_{0,1}^{\prime} h_{0,1}^{2}}\right)_{\sigma=\sigma_{\max }^{(0)}} \\
& \mathbb{H}_{r x} \Omega^{3}\left(\int_{\alpha(\sigma)}^{R} d \rho\left(\sum_{n=0}^{3} \mathcal{L}_{(n)}^{0 t h} \Omega^{n}\right)_{w_{0}}\right)_{\sigma=\sigma_{\max }^{(0)}} \\
& \sim \frac{\lambda}{\Omega}\left(-\frac{2 g_{3,3} \mathcal{E}^{2}}{h_{0,1}^{\prime} h_{0,1}^{2}}\right)_{\sigma=\sigma_{\max }^{(0)}} \\
& \mathbb{H}_{r x} \Omega^{3}\left(\frac{\sqrt{h_{0,1}^{\prime}(\sigma)^{2}+1}\left(\log (R)-\log \left(\frac{\mathcal{E}}{h_{0,1}(\sigma)}\right)\right)}{h_{0,1}(\sigma)^{2}}\right)_{\sigma=\sigma_{\max }^{(0)}} \\
& =\mathcal{O}\left(\Omega^{3}\right) \text {, }
\end{aligned}
$$

because $h_{0,1}\left(\sigma_{\max }^{(0)}\right)=R / \mathcal{E}+\mathcal{O}(\Omega)$. The last term in (22) is given by

$$
\begin{aligned}
- & \frac{\lambda}{\Omega} \int_{-\sigma_{\max }^{(0)}}^{\sigma_{\max }^{(0)}} d \sigma \beta(\sigma)\left(\sum_{n=0}^{3} \mathcal{L}_{(n)}^{0 t h} \Omega^{n}\right)_{\rho=\alpha(\sigma)} \\
= & \left(-\frac{\lambda}{\Omega}\right) \mathcal{E}^{2} \int_{-\sigma_{\max }^{(0)}}^{\sigma_{\max }^{(0)}} d \sigma\left(-\frac{\mathbb{H}_{r x} \Omega^{3} g_{3,3}(\sigma) \sqrt{h_{0,1}^{\prime}(\sigma)^{2}+1}}{h_{0,1}(\sigma)^{5}}\right) \\
& +\mathcal{O}\left(\Omega^{3}\right),
\end{aligned}
$$

where the integration is finite and so this term is quadratic in $\epsilon$. We may ignore this.

\section{References}

1. S. Ryu, T. Takayanagi, Holographic derivation of entanglement entropy from AdS/CFT. Phys. Rev. Lett. 96, 181602 (2006). arXiv:hep-th/0603001

2. J.M. Maldacena, The Large $\mathrm{N}$ limit of superconformal field theories and supergravity. Int. J. Theor. Phys. 38, 1113 (1999)

3. J.M. Maldacena, The Large $\mathrm{N}$ limit of superconformal field theories and supergravity. Adv. Theor. Math. Phys. 2, 231 (1998). arXiv:hep-th/9711200

4. M. Van Raamsdonk, Comments on quantum gravity and entanglement, arXiv:0907.2939 [hep-th]

5. M. Van Raamsdonk, Building up spacetime with quantum entanglement. Gen. Rel. Grav. 42, 2323 (2010). arXiv:1005.3035 [hep-th]

6. M. Van Raamsdonk, Building up spacetime with quantum entanglement. Int. J. Mod. Phys. D 19, 2429 (2010)

7. I.R. Klebanov, D. Kutasov, A. Murugan, Entanglement as a probe of confinement. Nucl. Phys. B 796, 274 (2008). arXiv:0709.2140 [hep-th]

8. T. Albash, C.V. Johnson, Holographic Studies of Entanglement Entropy in Superconductors. JHEP 1205, 079 (2012). arXiv:1202.2605 [hep-th]

9. R.G. Cai, S. He, L. Li, Y.L. Zhang, Holographic Entanglement Entropy in Insulator/Superconductor Transition. JHEP 1207, 088 (2012). arXiv:1203.6620 [hep-th]

10. J. Abajo-Arrastia, J. Aparicio, E. Lopez, Holographic Evolution of Entanglement Entropy. JHEP 1011, 149 (2010). arXiv: 1006.4090 [hep-th]

11. T. Albash, C.V. Johnson, Evolution of holographic entanglement entropy after thermal and electromagnetic quenches. New J. Phys. 13, 045017 (2011). arXiv:1008.3027 [hep-th]

12. V. Balasubramanian et al., Holographic thermalization. Phys. Rev. D 84, 026010 (2011). arXiv:1103.2683 [hep-th]

13. V. Balasubramanian et al., Thermalization of strongly coupled field theories. Phys. Rev. Lett. 106, 191601 (2011). arXiv:1012.4753 [hep-th]

14. R.C. Myers, A. Sinha, Seeing a c-theorem with holography. Phys. Rev. D 82, 046006 (2010). arXiv:1006.1263 [hep-th]

15. R.C. Myers, A. Sinha, Holographic c-theorems in arbitrary dimensions. JHEP 1101, 125 (2011). arXiv:1011.5819 [hep-th]

16. P. Bueno, R.C. Myers, W. Witczak-Krempa, Universality of corner entanglement in conformal field theories. Phys. Rev. Lett. 115, 021602 (2015). arXiv:1505.04804 [hep-th]

17. P. Bueno, R.C. Myers, Corner contributions to holographic entanglement entropy. JHEP 1508, 068 (2015). arXiv:1505.07842 [hepth] 
18. C. Park, Logarithmic Corrections to the Entanglement Entropy. Phys. Rev. D 92(12), 126013 (2015). arXiv:1505.03951 [hep-th]

19. K.K. Kim, O.K. Kwon, C. Park, H. Shin, Renormalized entanglement entropy flow in mass-deformed ABJM theory. Phys. Rev. D 90(4), 046006 (2014). arXiv:1404.1044 [hep-th]

20. K.K. Kim, O.K. Kwon, C. Park, H. Shin, Holographic entanglement entropy of mass-deformed Aharony-Bergman-Jafferis-Maldacena theory. Phys. Rev. D 90(12), 126003 (2014). arXiv:1407.6511 [hepth]

21. C. Kim, K.K. Kim, O.K. Kwon, Holographic Entanglement Entropy of Anisotropic Minimal Surfaces in LLM Geometries. Phys. Lett. B 759, 395 (2016). arXiv:1605.00849 [hep-th]

22. D. Jang, Y. Kim, O.K. Kwon, D.D. Tolla, Exact Holography of the Mass-deformed M2-brane Theory. Eur. Phys. J. C 77(5), 342 (2017). arXiv:1610.01490 [hep-th]

23. D. Jang, Y. Kim, O.K. Kwon, D.D. Tolla, Mass-deformed ABJM theory and LLM geometries: exact holography. JHEP 1704, 104 (2017). arXiv:1612.05066 [hep-th]

24. O.K. Kwon, D. Jang, Y. Kim, D.D. Tolla, Gravity from entanglement and RG flow in a top-down approach. JHEP 1805, 009 (2018). arXiv:1712.09101 [hep-th]

25. D. Jang, Y. Kim, O.K. Kwon, D.D. Tolla, Exact holography of massive M2-brane theories and entanglement entropy. EPJ Web Conf. 168, 07002 (2018)

26. G.T. Horowitz, J.E. Santos, D. Tong, Optical conductivity with holographic lattices. JHEP 1207, 168 (2012). arXiv:1204.0519 [hep-th]

27. A. Donos, S.A. Hartnoll, Interaction-driven localization in holography. Nature Phys. 9, 649 (2013). arXiv:1212.2998 [hep-th]

28. T. Andrade, B. Withers, A simple holographic model of momentum relaxation. JHEP 1405, 101 (2014). arXiv:1311.5157 [hep-th]

29. D. Vegh, Holography without translational symmetry. arXiv:1301.0537 [hep-th]

30. G.T. Horowitz, J.E. Santos, General relativity and the cuprates. JHEP 1306, 087 (2013). arXiv:1302.6586 [hep-th]

31. M. Blake, D. Tong, Universal resistivity from holographic massive gravity. Phys. Rev. D 88(10), 106004 (2013). arXiv: 1308.4970 [hep-th]

32. R.A. Davison, Momentum relaxation in holographic massive gravity. Phys. Rev. D 88, 086003 (2013). arXiv:1306.5792 [hep-th]

33. R.A. Davison, K. Schalm, J. Zaanen, Holographic duality and the resistivity of strange metals. Phys. Rev. B 89(24), 245116 (2014). arXiv:1311.2451 [hep-th]

34. A. Donos, J.P. Gauntlett, Holographic Q-lattices. JHEP 1404, 040 (2014). arXiv:1311.3292 [hep-th]

35. M. Blake, D. Tong, D. Vegh, Holographic Lattices Give the Graviton an Effective Mass. Phys. Rev. Lett. 112(7), 071602 (2014). arXiv:1310.3832 [hep-th]

36. A. Donos, J.P. Gauntlett, Novel metals and insulators from holography. JHEP 1406, 007 (2014). arXiv: 1401.5077 [hep-th]

37. K.Y. Kim, K.K. Kim, Y. Seo, S.J. Sin, Coherent/incoherent metal transition in a holographic model. JHEP 1412, 170 (2014). arXiv: 1409.8346 [hep-th]

38. B. Gouteraux, Charge transport in holography with momentum dissipation. JHEP 1404, 181 (2014). arXiv:1401.5436 [hep-th]

39. M. Blake, A. Donos, Quantum Critical Transport and the Hall Angle. Phys. Rev. Lett. 114(2), 021601 (2015). arXiv:1406.1659 [hep-th]

40. A. Donos, J.P. Gauntlett, Thermoelectric DC conductivities from black hole horizons. JHEP 1411, 081 (2014). arXiv:1406.4742 [hep-th]

41. K.Y. Kim, K.K. Kim, Y. Seo, S.J. Sin, Thermoelectric conductivities at finite magnetic field and the nernst effect. JHEP 1507, 027 (2015). arXiv:1502.05386 [hep-th]
42. M. Blake, A. Donos, N. Lohitsiri, Magnetothermoelectric response from holography. JHEP 1508, 124 (2015). arXiv:1502.03789 [hepth]

43. Y. Seo, K.Y. Kim, K.K. Kim, S.J. Sin, Character of matter in holography: spin-orbit interaction. Phys. Lett. B 759, 104 (2016). arXiv:1512.08916 [hep-th]

44. S. Khimphun, B.H. Lee, C. Park, Conductivities in an anisotropic medium. Phys. Rev. D 94(8), 086005 (2016). arXiv:1604.00156 [hep-th]

45. C. Park, On black hole thermodynamics with a momentum relaxation. Class. Quant. Grav. 33(24), 245017 (2016). arXiv:1606.07340 [hep-th]

46. Y. Seo, G. Song, P. Kim, S. Sachdev, S.J. Sin, Holography of the dirac fluid in graphene with two currents. Phys. Rev. Lett. 118(3), 036601 (2017). arXiv:1609.03582 [hep-th]

47. B. Withers, Nonlinear conductivity and the ringdown of currents in metallic holography. JHEP 1610, 008 (2016). arXiv:1606.03457 [hep-th]

48. R.A. Davison, W. Fu, A. Georges, Y. Gu, K. Jensen, S. Sachdev, Thermoelectric transport in disordered metals without quasiparticles: The Sachdev-Ye-Kitaev models and holography. Phys. Rev. B 9515, 155131 (2017). arXiv:1612.00849 [cond-mat.str-el]

49. A. O'Bannon, J. Probst, R. Rodgers, C.F. Uhlemann, First law of entanglement rates from holography. Phys. Rev. D 966, 066028 (2017). arXiv:1612.07769 [hep-th]

50. A. Bagrov, B. Craps, F. Galli, V. Keränen, E. Keski-Vakkuri, J. Zaanen, Holographic pump probe spectroscopy. JHEP 1807, 065 (2018). arXiv:1804.04735 [hep-th]

51. M. Reza Mohammadi Mozaffar, A. Mollabashi, F. Omidi, Nonlocal probes in holographic theories with momentum relaxation. JHEP 1610, 135 (2016). arXiv:1608.08781 [hep-th]

52. O. Aharony, O. Bergman, D.L. Jafferis, J. Maldacena, N=6 superconformal chern-simons-matter theories, M2-branes and their gravity duals. JHEP 0810, 091 (2008). arXiv:0806.1218 [hep-th]

53. N.I. Gushterov, A. O'Bannon, R. Rodgers, On holographic entanglement density. JHEP 1710, 137 (2017). arXiv:1708.09376 [hepth]

54. S.A. Hartnoll, P.K. Kovtun, M. Muller, S. Sachdev, Theory of the Nernst effect near quantum phase transitions in condensed matter, and in dyonic black holes. Phys. Rev. B 76, 144502 (2007). arXiv:0706.3215 [cond-mat.str-el]

55. S.A. Hartnoll, C.P. Herzog, Impure AdS/CFT correspondence. Phys. Rev. D 77, 106009 (2008). arXiv:0801.1693 [hep-th]

56. H. Liu, M. Mezei, A Refinement of entanglement entropy and the number of degrees of freedom. JHEP 1304, 162 (2013). arXiv: 1202.2070 [hep-th]

57. H. Casini, M. Huerta, On the RG running of the entanglement entropy of a circle. Phys. Rev. D 85, 125016 (2012). arXiv: 1202.5650 [hep-th]

58. T. Nishioka, Entanglement entropy: holography and renormalization group. Rev. Mod. Phys. 90(3), 035007 (2018). arXiv:1801.10352 [hep-th]

59. S.A. Hartnoll, C.P. Herzog, Ohm's Law at strong coupling: S duality and the cyclotron resonance. Phys. Rev. D 76, 106012 (2007). arXiv:0706.3228 [hep-th]

60. K. Bidzhiev, G. Misguich, Out-of-equilibrium dynamics in a quantum impurity model: Numerics for particle transport and entanglement entropy. Phys. Rev. B 96(19), 195117 (2017). arXiv:1707.06111 [cond-mat.str-el]

61. N. Laflorencie, Quantum entanglement in condensed matter systems. Phys. Rept. 646, 1 (2016). arXiv:1512.03388 [cond-mat.strel]

62. S.F. Lokhande, G.W.J. Oling, J.F. Pedraza, JHEP 1710, 104 (2017). https://doi.org/10.1007/JHEP10(2017). arXiv:1705.10324 [hep-th] 
63. J. Bhattacharya, M. Nozaki, T. Takayanagi, T. Ugajin, Phys. Rev. Lett. 110(9), 091602 (2013). https://doi.org/10.1103/PhysRevLett. 110.091602. arXiv:1212.1164 [hep-th]

64. D.D. Blanco, H. Casini, L.Y. Hung, R.C. Myers, JHEP 1308, 060 (2013). https://doi.org/10.1007/JHEP08(2013). arXiv:1305.3182 [hep-th]

65. G.T. Horowitz, N. Iqbal, J.E. Santos, Phys. Rev. D 88(12), 126002 (2013). https://doi.org/10.1103/PhysRevD.88.126002. arXiv: 1309.5088 [hep-th]

66. D. Bak, S. Yun, Thermal aspects of ABJM theory: currents and condensations. Class. Quant. Grav. 27, 215011 (2010). arXiv: 1001.4089 [hep-th]

67. D. Bak, K.K. Kim, S. Yun, Symmetry breaking phase transitions in ABJM theory with a finite U(1) chemical potential. Phys. Rev. D 84, 086010 (2011). arXiv:1011.5749 [hep-th]

68. T. Faulkner, R.G. Leigh, O. Parrikar, Shape dependence of entanglement entropy in conformal field theories. JHEP 1604, 088 (2016). arXiv:1511.05179 [hep-th]

69. R.X. Miao, A holographic proof of the universality of corner entanglement for CFTs. JHEP 1510, 038 (2015). arXiv:1507.06283 [hep-th]

70. P. Bueno, R.C. Myers, W. Witczak-Krempa, Universal corner entanglement from twist operators. JHEP 1509, 091 (2015). arXiv: 1507.06997 [hep-th]

71. L.E.H. Sierens, P. Bueno, R.R.P. Singh, R.C. Myers, R.G. Melko, Cubic trihedral corner entanglement for a free scalar. Phys. Rev. B 96(3), 035117 (2017). arXiv:1703.03413 [cond-mat.str-el]
72. H. Osborn, A.C. Petkou, Implications of conformal invariance in field theories for general dimensions. Annals Phys. 231, 311 (1994). arXiv:hep-th/9307010

73. R. Islam, R. Ma, P .M. Preiss, M .E. Tai, A. Lukin, M. Rispoli, M. Greiner. Measuring entanglement entropy in a quantum many-body system. Nature 528, 77-83

74. A.K. Ekert, C.M. Alves, D.K.L. Oi, M. Horodecki, P. Horodecki, L.C. Kwek, Direct estimations of linear and nonlinear functionals of a quantum state. Phys. Rev. Lett. 88, 217901 (2002)

75. C.M. Alves, D. Jaksch, Multipartite entanglement detection in bosons. Phys. Rev. Lett. 93, 110501 (2004)

76. Y. Wang, L. Li, N.P. Ong, The nernst effect in high- $t_{c}$ superconductors. Phys. Rev. B 73, 024510 (2006). arXiv:cond-mat/0510470

77. H.F. Song, S. Rachel, C. Flindt, I. Klich, N. Laflorencie, K.L. Hur, Bipartite fluctuations as a probe of many-body entanglement. Phys. Rev. B 85, 035409 (2012) 